This is an Open Access article, distributed under the terms of the Creative Commons Attribution licence (http://creativecommons.org/licenses/by/4.0/), which permits unrestricted re-use, distribution, and reproduction in any medium, provided the original work is properly cited. doi:10.1017/jfm.2020.959

\title{
Coriolis effect on centrifugal buoyancy-driven convection in a thin cylindrical shell
}

\author{
Amirreza Rouhi ${ }^{1,2, \dagger}$, Detlef Lohse ${ }^{3,4}$, Ivan Marusic ${ }^{2}$, Chao Sun ${ }^{3,4}$ and \\ Daniel Chung ${ }^{2}$
}

${ }^{1}$ Department of Engineering, School of Science and Technology, Nottingham Trent University, Nottingham NG11 8NS, UK

${ }^{2}$ Department of Mechanical Engineering, University of Melbourne, Victoria 3010, Australia

${ }^{3}$ Physics of Fluids Group and Max Planck Center Twente,

MESA+ Institute and J. M. Burgers Centre for Fluid Dynamics, University of Twente, P.O. Box 217, 7500AE Enschede, The Netherlands

\footnotetext{
${ }^{4}$ Department of Energy and Power Engineering, Center for Combustion Energy, Key Laboratory for Thermal Science and Power Engineering of Ministry of Education, International Joint Laboratory on Low Carbon Clean Energy Innovation, Tsinghua University, Beijing 100084, PR China
}

(Received 17 February 2020; revised 16 October 2020; accepted 26 October 2020)

We study the effect of the Coriolis force on centrifugal buoyancy-driven convection in a rotating cylindrical shell with inner cold wall and outer hot wall. This is done by performing direct numerical simulations for increasing inverse Rossby number $R o^{-1}$ from zero (no Coriolis force) to 20 (very large Coriolis force) and for Rayleigh number $R a$ from $10^{7}$ to $10^{10}$ and Prandtl number $\operatorname{Pr}=0.7$, corresponding to air. We invoke the thin-shell limit, which neglects the curvature and radial variations of the centripetal acceleration. As $R o^{-1}$ increases from zero, the system forms an azimuthal bidirectional wind that reaches its maximum momentum at an optimal $R o_{o p t}^{-1}$, associated with a maximal skin-friction coefficient $C_{f}$ and a minimal Nusselt number $N u$. Just beyond $R o_{o p t}^{-1}$, the wind weakens and an axial, quasi-two-dimensional cyclone, corotating with the system, begins to form. A local 'turbulence' inverse Rossby number (non-dimensionalised by the eddy turnover time) determines the onset of cyclone formation for all $R a$, when its value reaches approximately 4. At $R o^{-1} \gg R o_{o p t}^{-1}$, the system falls into the geostrophic regime with a sudden drop in $\mathrm{Nu}$. The bidirectional wind for $R o^{-1} \leq R o_{o p t}^{-1}$ is a feature of this system, as it hastens the boundary layer transition from laminar to turbulent, towards the ultimate regime. We see the onset of this transition at $R a=10^{10}$ and $R o^{-1} \simeq R o_{\text {opt }}^{-1}$, although the mean flow profile has not yet fully collapsed on the Prandtl-von Kármán (logarithmic) law.

Key words: Bénard convection, rotating turbulence, turbulence simulation

$†$ Email address for correspondence: amirreza.rouhi@ntu.ac.uk 


\section{Introduction}

Thermal convection is an important transport mechanism in many engineering and geophysical flows. Centrifugal buoyancy-driven convection (figure 1) is a canonical thermal convection system to study some of these flows (table 1). The studies with geophysical interests consider this system as a closed dynamical model for the earth's liquid (outer) core (Busse \& Carrigan 1974), or midlatitude atmosphere (Randriamampianina et al. 2006; Read et al. 2008; Von Larcher et al. 2018). The studies with turbomachinery interests consider this system as a model for the compressor cavity (Bohn et al. 1995; King, Wilson \& Owen 2007; Owen \& Long 2015; Pitz et al. 2017a; Pitz, Marxen \& Chew 2017b). The system is a rotating cylindrical shell with inner cold wall and outer hot wall (figure 1). Rotation introduces centrifugal buoyancy (set by centripetal acceleration) and Coriolis forces.

Centrifugal convection differs from rotating Rayleigh-Bénard convection (table 1). In centrifugal convection, the axis of rotation is parallel to the hot and cold walls (normal to the centrifugal buoyancy force), while in rotating Rayleigh-Bénard convection, the axis of rotation is normal to the hot and cold walls (parallel to the gravitational buoyancy force). However, both systems are characterised by the Rayleigh number $R a$, inverse Rossby number $R_{o}^{-1}$ and Prandtl number $\operatorname{Pr}$ (assuming that gravity is neglected in centrifugal convection, figure $1 a$ ). In centrifugal convection, these numbers are defined as

$$
\begin{gathered}
R a \equiv(U H)^{2} /(\nu \kappa)=\left(\Omega^{2} R \beta \Delta H^{3}\right) /(\nu \kappa), \\
R o^{-1} \equiv(2 \Omega H) / U=2(\beta \Delta R / H)^{-1 / 2}, \\
P r \equiv v / \kappa,
\end{gathered}
$$

where $U \equiv\left(\Omega^{2} R \beta \Delta H\right)^{1 / 2}$, the free-fall velocity; $\Omega$ is the rotational speed; $R$ is the outer shell radius; $H$ is the shell thickness; $\Delta \equiv\left(T_{H}-T_{L}\right)$, the temperature difference; $\beta$ is the thermal expansion coefficient; $\kappa$ is the thermal diffusivity; $v$ is the kinematic viscosity. For convenience, we only use the inverse Rossby number $R o^{-1}$ (rather than $R o$ ), as it is directly linked to the Coriolis force (i.e. higher $\mathrm{Ro}^{-1}$ implies higher Coriolis force).

In figure 2, we compile a $\left(R a, R o^{-1}\right)$ parameter space of the previous studies on centrifugal convection (figure $2 a$ ) and rotating Rayleigh-Bénard convection (figure $2 b$ ). We list these studies in table 1 . We also add two recent sets of data points for centrifugal convection (figure $2 a$ ). One is our present data ( $\circ$, black) and the other one is by our coauthor, Professor C. Sun and his colleagues $(\diamond$, dark grey $\diamond$, process blue $\diamond$, dark orange; Jiang et al. (2020)). This figure highlights the importance of studying centrifugal convection, as a system that is explored to a lesser extent than rotating Rayleigh-Bénard convection. Rotating Rayleigh-Bénard convection has been investigated over almost a continuous parameter sweep of $10^{4} \lesssim R a \lesssim 10^{15}$ and $0 \lesssim R o^{-1} \lesssim 100$. However, until recent studies ( $\circ$, black; $\diamond$, dark grey; $\diamond$, process blue; $\diamond$, dark orange), centrifugal convection was investigated at limited values of $R a$ and $R o^{-1}$, and only for $R o^{-1} \gtrsim 2$ (large Coriolis force). von Hardenberg et al. (2015) study $R o^{-1}<2$ ( $\nabla$, red), but they consider $R a=10^{7}$. Also, their set-up is free-slip hot and cold plates rotating about a distant axis. This set-up can be perceived as a slice of a thin cylindrical shell with free-slip boundaries (CC_slip in table 1). The previous studies focus on large $\mathrm{Ro}^{-1}$ because experiments are constrained by their working fluid and apparatus, and numerical studies set their parameter space following the experiments. For instance, among the geophysical studies, Read et al. (2008) ( $\triangle$, blue) follow the experiment of Fowlis \& Hide (1965), and Von Larcher et al. (2018) ( $\triangle$, black) follow their own experiment. Among the turbomachinery studies, 

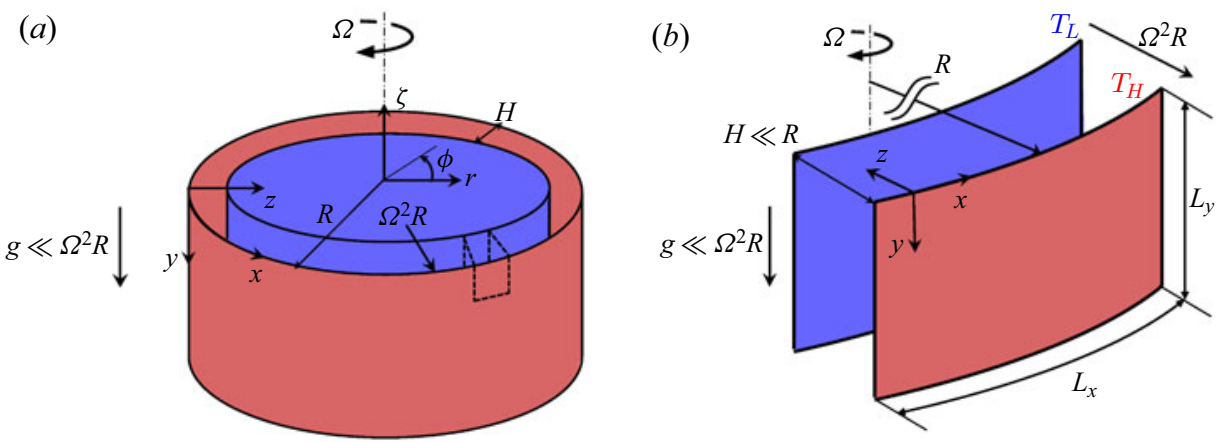

FIGURE 1. Set-up of flow. (a) Centrifugal buoyancy-driven convection in the cylindrical shell with gap $H$ and outer radius $R$. The shell undergoes solid-body clockwise rotation about its axis $\zeta$, with rotational speed $\Omega$. The outer wall is hotter than the inner core. $(b)$ Our computational domain as a small chunk of centrifugal convection, highlighted with dashed lines in panel $(a)$, with $H \ll R$, which is rectilinear, and $L_{x}$ and $L_{y}$ are the domain sizes in the streamwise (circumferential) and spanwise (axial) directions. Due to thin-shell approximation, the computational domain is exposed to a uniform centripetal acceleration $\Omega^{2} R \gg g$, so that gravity does not matter here.

Pitz et al. (2017a,b) ( $\square$, red) and King et al. (2007) ( $\square$, black) follow the experiment of Bohn et al. (1995) ( $\square$, blue).

Here, we perform DNS at $R a=\left(10^{7}, 10^{8}, 10^{9}, 10^{10}\right)$ and $R o^{-1}=(0,0.3,0.5,0.6,0.8$, $1.0)$, (o, black in figure 2a). Additionally, we explore $\left(R a, R o^{-1}\right)=\left(10^{7}, 2.0\right)$ and $\left(R a, R o^{-1}\right)=\left(10^{8}, 20.0\right)$ that fall into the regimes of interest by the geophysical and turbomachinery studies. Our objectives are to investigate: (i) the flow regimes from no Coriolis force $\left(R o^{-1}=0\right)$ to a very large Coriolis force $\left(R o^{-1}=20\right)$; (ii) the universality of these regimes from $R a=10^{7}$ to $10^{10}$; and (iii) the analogy between these regimes and those in rotating Rayleigh-Bénard convection. We show how an optimal choice of $R o^{-1}$ can exploit the Coriolis force to tune a persistent large-scale wind. On the other hand, we show how large $R o^{-1} \geq 1$ can cause the Coriolis force to suppress turbulence and laminarise the flow. The organised wind is a feature of this system. It hastens the boundary layer transition from laminar to turbulent, i.e. transition from the classical regime (Grossmann \& Lohse 2000) to the ultimate regime (Grossmann \& Lohse 2011). In the classical regime, the effective heat-transfer scaling, expressed through an effective power law for the Nusselt number $N u$ to $R a$ relationship $\left(N u \propto R a^{\alpha}\right)$, follows an effective power-law exponent $\alpha \leq 1 / 3$ (Grossmann \& Lohse 2000). However, in the ultimate regime the heat-transfer scaling follows a steeper gradient with an effective exponent $\alpha>1 / 3$ (Grossmann \& Lohse 2011).

The ultimate regime has been approached or fully reached in several turbulent systems, including Rayleigh-Bénard convection (Roche et al. 2005; Ahlers et al. 2012; He et al. 2012a,b, 2013; He, Bodenschatz \& Ahlers 2016), the analogue Taylor-Couette flow (see the review by Grossmann, Lohse \& Sun (2016)), and vertical natural convection (Ng et al. 2017) or sheared convection (Pirozzoli et al. (2017) and Blass et al. (2020), though by far not reached here). The three latter systems benefit from a persistent wind, similar to centrifugal convection. However, the source of wind formation, i.e. shear, differs among these systems; in Taylor-Couette flow the shear is due to differentially rotating cylinders, in sheared convection the shear is due to differentially moving walls, in vertical convection the shear is due to gravitational buoyancy, and in centrifugal convection the shear is due 


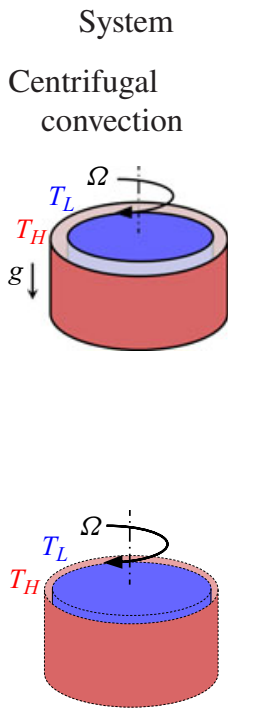

Rotating rot_RB_wall
Rayleigh-Bénard
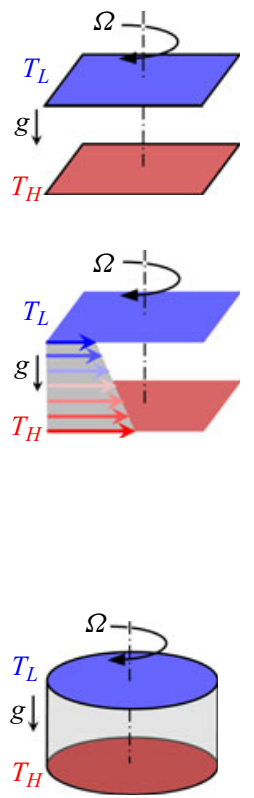

CC_wall

$$
\text { CC_slip }
$$

Name Description

A cylindrical shell with outer hot wall and inner cold wall

Similar to

CC_wall but in a thin shell with free-slip hot and cold boundaries

\section{A horizontally unbounded domain with lower hot wall and upper cold wall}

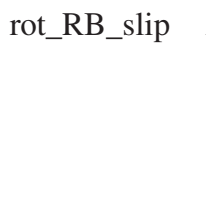

A horizontally
unbounded
domain with an
imposed
temperature
gradient and
free-slip
condition at the
top and bottom
A cylindrical cell
with lower hot
wall, upper cold
wall and
adiabatic
sidewall

Julien et al. (1996a,b, 1999)

Kunnen et al. (2016)

Favier et al. (2014)

Guervilly et al. (2014)

Novi et al. (2019)

Vorobieff \& Ecke (2002)

Ecke \& Niemela (2014)

King et al. (2009)

Cheng et al. (2015)

Stevens et al. $(2009,2011,2012)$

Kunnen et al. (2008, 2010a,b, 2011)

Horn \& Shishkina $(2014,2015)$

Weiss \& Ahlers (2011a)

Weiss et al. $(2010,2016)$

Zhong \& Ahlers (2010)

Zhong et al. (2017)

Zhang et al. (2020)

de Wit et al. (2020)

TABLE 1. Schematic representation and previous studies of centrifugal convection and rotating Rayleigh-Bénard convection. We name the systems based on their boundary conditions (wall or slip). 

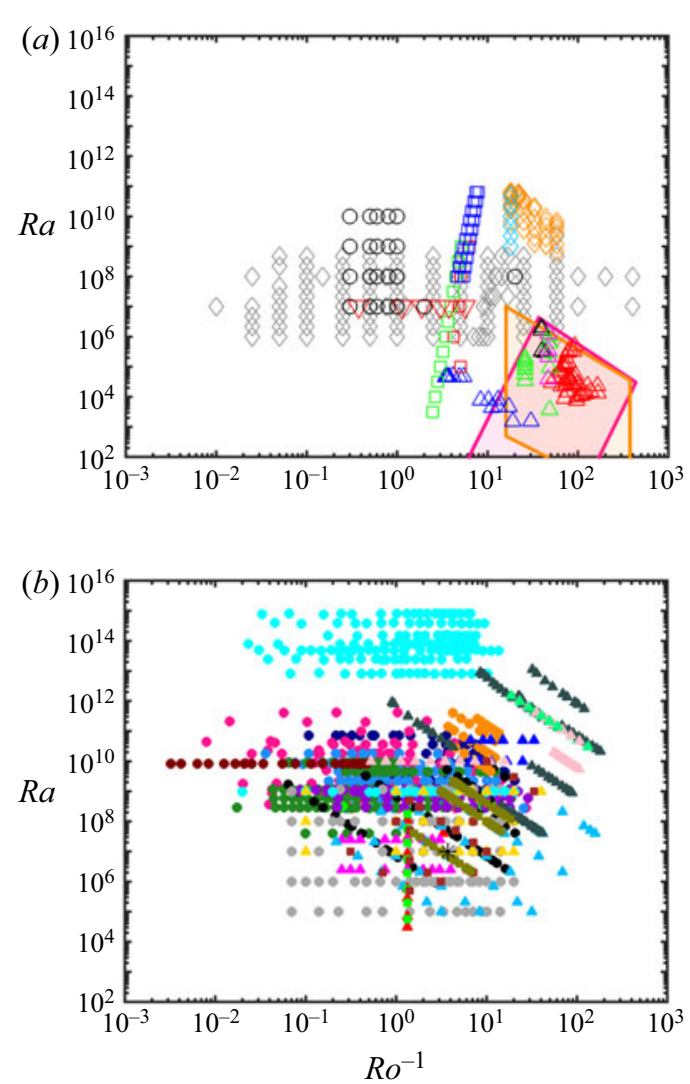

rot_RB_slip

Julien et al. (1996a,b, 1999)

$\Delta$ Kunnen et al. (2016)

Favier et al. (2014)

Guervilly et al. (2014)
CC_wall

- Present data

Fowlis \& Hide (1965)

Hide \& Mason (1970)

$\triangle$ Busse \& Carrigan (1974)

$\triangle$ Read et al. (2008)

$\triangle$ Von Larcher \& Egbers (2005)

$\triangle$ Von Larcher \& Dörnbrack (2014)

$\triangle$ Von Larcher et al. (2018)

口 Bohn et al. (1995)

ㅁ Pitz et al. $(2017 a, b)$

$\square$ King et al. (2007)

$\diamond \diamond \diamond$ Jiang et al. (2020)

CC_slip

$\nabla$ von Hardenberg et al. (2015)

rot_RB_wall

A Julien et al. (1996a,b, 1999)

$\Delta$ Kunnen et al. (2006, 2009)

$\Delta$ Kunnen et al. (2016)

$\Delta$ King et al. $(2009,2012)$

$\Delta$ Cheng et al. (2015)

Chong et al. (2017)

$\Delta$ de Wit et al. (2020)

$\triangle$ Aguirre Guzmán et al. (2020)

rot_RB_cyl

- King et al. $(2009,2012)$

- Vorobieff \& Ecke (2002)

- Ecke \& Niemela (2014)

- Stevens et al. $(2009,2011,2012)$

- Kunnen et al. $(2008,2010 a, b, 2011)$

- Horn \& Shishkina $(2014,2015)$

- Weiss \& Ahlers (2011a)

- Weiss et al. (2010, 2016)

- Zhong \& Ahlers (2010)

- Zhong et al. (2017)

$\Delta$ Cheng et al. (2015)

- Zhang et al. (2020)

$\Delta$ de Wit et al. (2020)

FIGURE 2. Parameter space of $R a$ and $R o^{-1}$ corresponding to our present simulations (o, black in panel $a$ ) and the previous studies listed in table 1 for $(a)$ centrifugal convection (shaded regions or open symbols) and $(b)$ rotating Rayleigh-Bénard convection (filled symbols). For centrifugal convection, the data of Jiang et al. (2020) are divided between three-dimensional direct numerical simulations (DNS) ( $\diamond$, dark grey), two-dimensional DNS ( $\diamond$, process blue) and experiments $(\diamond$, dark orange). Shaded regions or open triangles correspond to the studies with geophysical interests, and open squares correspond to the studies with turbomachinery interests. The shaded regions highlight the approximate parameter space, because the exact $\left(R o^{-1}, R a\right)$ values are not reported. For rotating Rayleigh-Bénard convection, the symbol shape is different between rot_RB_wall (filled triangle), rot_RB_slip (filled square) and rot_RB_cyl (filled circle). For centrifugal convection, $R a$ and $R o^{-1}$ are defined based on $(1.1 a)$ and $(1.1 b)$, respectively, where for the studies with finite shell thickness we pick $R$ as the average of inner core and outer wall radius. For rotating Rayleigh-Bénard convection, $R a \equiv \beta g \Delta H^{3} /(\kappa v)$ and $R o^{-1} \equiv 2 \Omega(g \beta \Delta / H)^{-1 / 2}$, where $g$ is gravity and $\beta, \Delta, \Omega, v, \kappa$ are the same as those in (1.1a)-(1.1c) and $H$ is the height of the sample between the top (cold) wall and bottom (hot) wall. 
to the Coriolis force. Centrifugal convection is unique among these systems, because the wind forms due to the Coriolis force that does not alter the kinetic energy. Our coauthor, Professor C. Sun, has proposed this system as an ideal opportunity to reach the ultimate turbulent regime, to mitigate possible non-Oberbeck-Boussinesq effects at large $R a$ in the standard Rayleigh-Bénard geometry, as here the possible ultimate regime is now triggered by centrifugal buoyancy instead of by temperature differences. It was this proposition which triggered the present numerical work.

The paper is organised as follows. In $\S 2$ we describe our DNS set-up as well as simulation and grid convergence studies. In the results section $(\S 3)$, first, we identify the overall flow regimes ( $\$ 3.1$ ) through flow visualisation, $N u$ and skin-friction coefficient $C_{f}$. Then, we discuss each flow regime from the bidirectional wind formation $(\S 3.2)$ to the flow laminarisation $(\S 3.5)$. In $\S 3.6$ we show the onset of transition to turbulent by the bidirectional wind. The concluding remarks follow in $\S 4$.

\section{Flow set-up}

\subsection{Governing equations}

The governing equations are derived from the incompressible Navier-Stokes equations governing the flow in a concentric cylindrical annulus with gap $H$ (figure $1 a$ ) in the frame rotating in a clockwise direction about its cylindrical axis $\zeta$ at constant rotational speed $\Omega$, as described by velocity $\boldsymbol{v}=v_{r} \boldsymbol{e}_{r}+v_{\phi} \boldsymbol{e}_{\phi}+v_{\zeta} \boldsymbol{e}_{\zeta}$ and temperature $T$ in cylindrical coordinates $(r, \phi, \zeta)$. The boundary conditions in this rotating frame are no-slip and impermeable walls, $\boldsymbol{v}(r=R-H)=\boldsymbol{v}(r=R)=0$, corresponding to the inner core and outer wall, respectively, and isothermal walls with the prescribed temperature difference $\Delta=T_{H}-T_{L}$, with $T(r=R-H)=T_{L}$ and $T(r=R)=T_{H}$, corresponding to an inner colder core and an outer hotter wall. We have invoked the Oberbeck-Boussinesq approximation, which assumes constant fluid properties, $v, \kappa$ and $\beta$, and that density variations are only dynamically important in the buoyancy term. In the buoyancy term the density variation is $\left(\rho-\rho_{o}\right)=-\beta \rho_{o} \theta$, where $\rho_{o}=\rho\left(T_{o}=\left(T_{H}+T_{L}\right) / 2\right)$, the reference density at temperature $T_{o}$, and $\theta=T-T_{o}$, the temperature variation relative to $T_{o}$. For the sake of brevity we refer the reader to Kundu \& Cohen (1990), for example, for the equations in the $(r, \phi, \zeta)$ coordinate system. Since the equations are presented in atating frame, two additional terms appear in the Navier-Stokes equations: the Coriolis force, $-2 \Omega v_{\phi} \boldsymbol{e}_{r}+2 \Omega v_{r} \boldsymbol{e}_{\phi}$, and the centripetal acceleration, $-\beta \Omega^{2} r \theta \boldsymbol{e}_{r}$.

To further simplify the problem, we consider the thin-shell (unity radius ratio) limit, $\epsilon \equiv H / R \ll 1$ (figure $1 b$ ). To this end, we transform the equations from $(r, \phi, \zeta)$ into curvilinear coordinates $(x, y, z)$ with the origin placed at the outer cylinder. The transformed coordinates will be $x=r \phi, y=-\zeta, z=R-r$, and the transformed velocity will be $u=v_{\phi}, v=-v_{\zeta}, w=-v_{r}$. Then, we non-dimensionalise the variables using the gap width $H$, the free-fall velocity $U \equiv\left(\Omega^{2} R \beta \Delta H\right)^{1 / 2}$, and $\Delta: \tilde{x}=x / H, \tilde{y}=y / H, \tilde{z}=$ $z / H, \tilde{t}=t U / H$ are the scaled space and time coordinates; $\tilde{u}=u / U, \tilde{v}=v / U, \tilde{w}=w / U$ are the scaled velocity components; and $\tilde{p}=\left(p-\rho_{o} \Omega^{2} R^{2} / 2\right) /\left(\rho_{o} U^{2}\right)$ and $\tilde{\theta}=\theta / \Delta$ are the scaled pressure and temperature variation. Substituting these into the transformed equation, and expanding in small $\epsilon$, we obtain, to leading order

$$
\begin{gathered}
\tilde{\nabla} \cdot \tilde{\boldsymbol{u}}=0, \\
\partial_{\tilde{t}} \tilde{\boldsymbol{u}}+\tilde{\boldsymbol{u}} \cdot \tilde{\nabla} \tilde{u}=-\partial_{\tilde{x}} \tilde{p}+(\operatorname{Ra} / \operatorname{Pr})^{-1 / 2} \tilde{\nabla}^{2} \tilde{u}-\operatorname{Ro}^{-1} \tilde{w}, \\
\partial_{\tilde{t}} \tilde{\boldsymbol{v}}+\tilde{\boldsymbol{u}} \cdot \tilde{\nabla} \tilde{v}=-\partial_{\tilde{y}} \tilde{p}+(\operatorname{Ra} / \operatorname{Pr})^{-1 / 2} \tilde{\nabla}^{2} \tilde{v},
\end{gathered}
$$




$$
\begin{gathered}
\partial_{t} \tilde{\boldsymbol{w}}+\tilde{\boldsymbol{u}} \cdot \tilde{\nabla} \tilde{\boldsymbol{w}}=-\partial_{\tilde{z}} \tilde{\boldsymbol{p}}+(R a / P r)^{-1 / 2} \tilde{\nabla}^{2} \tilde{\boldsymbol{w}}+R o^{-1} \tilde{\boldsymbol{u}}+\tilde{\theta}, \\
\partial_{\bar{t}} \tilde{\theta}+\tilde{\boldsymbol{u}} \cdot \tilde{\nabla} \tilde{\theta}=(R a P r)^{-1 / 2} \tilde{\nabla}^{2} \tilde{\theta} .
\end{gathered}
$$

In (2.1a)-(2.1e), the Rayleigh number $R a$, the inverse Rossby number $R o^{-1}$ and the Prandtl number $P r$, as introduced in $(1.1 a)-(1.1 c)$, are the control parameters. Since $\tilde{x}=O(1)$ and $\tilde{y}=O(1)$, the thin-shell limit implies $x \ll R$ and $y \ll R$, i.e. the computational domain is a small chunk of the concentric cylinder (indicated with dashed lines in figure 1a). Therefore, the transformed (2.1a)-(2.1e) that are identical to the Navier-Stokes equations in the Cartesian coordinate system are solved in a rectilinear box (figure $1 b$ ). The transformed boundary conditions in the $x$ - and $y$-directions are periodic and at the outer and inner walls are $\tilde{\boldsymbol{u}}(\tilde{z}=0)=\tilde{\boldsymbol{u}}(\tilde{z}=1)=0, \tilde{\theta}(\tilde{z}=0)=1 / 2$ and $\tilde{\theta}(\tilde{z}=1)=-1 / 2$.

The results are presented in terms of $(x, u),(z, w)$ and $(y, v)$, the circumferential, (negative) radial and (negative) axial directions of the cylindrical shell, respectively, and are noted as the streamwise, wall-normal and spanwise directions. The inner and outer walls of the cylindrical shell are also noted as the top and bottom walls, respectively. In the entire manuscript, we denote a non-dimensionalised quantity by $U$ and $H$ with tilde (e.g. $\tilde{t}=t U / H)$, an $x y$-plane and time averaged quantity with overbar (e.g. $\bar{u}$ ), a volume and time averaged quantity with angle bracket (e.g. $\langle u\rangle)$ and an averaged quantity over a specific direction with superscript (e.g. $u^{y}$ is averaged in the $y$-direction). Fluctuating quantities are obtained by subtracting $x y$-plane and time averaged quantities from their instantaneous counterpart (e.g. $u^{\prime}=u-\bar{u}$ ).

\section{2. $D N S$}

The equations are solved using a fully conservative fourth-order finite-difference code, validated in the previous DNS studies of similar flow physics ( $\mathrm{Ng}$ et al. 2015, 2017, 2018). Table 2 lists all the simulation cases and figure 3 shows the visualizations of the production runs. For all cases, $\operatorname{Pr}=0.7$ and $L_{x} / H \times L_{y} / H=1 \times 1$. We choose a fixed aspect ratio of $\Gamma=1$ to focus on the Coriolis force effect $\left(R o^{-1}\right)$ and achieve the highest possible $R a$. Nevertheless, we speculate that the essential physics, hence the trend in $\mathrm{Nu}$ and skin-friction coefficient $C_{f}$ do not change with $\Gamma$. Our conjecture is based on the previous studies on centrifugal convection and similar thermal convection systems. In classical Rayleigh-Bénard convection, the sensitivity of $N u$ with $\Gamma \geq 1$ is less than $7 \%$ for $R a \gtrsim 2 \times 10^{7}$ (figure $4 a$ in Stevens et al. (2018)). The sensitivity of $\alpha\left(N u \propto R a^{\alpha}\right)$ with $\Gamma \geq 1$ is less than $3 \%$ for $R a \gtrsim 10^{9}$ (we analysed figure 3 of Zhou et al. (2012)). In rotating Rayleigh-Bénard convection, $N u$ is almost insensitive to $\Gamma \geq 1$ for $R o^{-1} \geq 0.4$ (figure 4 in Stevens et al. (2011)). Even for $R o^{-1}<0.4, N u$ versus $R o^{-1}$ shows the same trend for different $\Gamma$. In centrifugal convection, the trend in $N u$ versus $R o^{-1}$ for a full cylindrical shell (figure $2 a$ in Jiang et al. (2020)) is similar to what we report in $\S 3.1$. Also, the flow regimes that we discuss in $\S 3$ are similar to the previous experiments. At low rotational speed, experiments report an axisymmetric flow, circulating parallel to the walls (e.g. figure $2 a, b$ in Fowlis \& Hide (1965); figure 13e, $f$ in Hide \& Mason (1970)). We observe similar flow regime (bidirectional wind) in §3.2. At high rotational speed, experiments report geostrophic regime with large-scale quasi-two-dimensional cyclones and anticyclones (e.g. figure $2 d-h$ in Fowlis \& Hide (1965); figure 4 in Jiang et al. (2020)). We observe similar flow regime in $\S 3.5$.

We use the same number of grid points $N$ in each direction. The grid points are uniformly distributed in the $x$ - and $y$-directions, and are stretched in the $z$-direction. The choice for $N$ and stretching factor are decided a priori based on Shishkina et al. (2010, 


\begin{tabular}{|c|c|c|c|c|c|c|c|c|c|c|c|}
\hline & $R o^{-1}$ & $\tilde{\tau}_{\text {avg }}$ & $\mathrm{Nu}$ & $N u_{h}$ & $N u_{b o t}$ & $\operatorname{Diff}_{\tau_{\text {avg }}}$ & $N_{B L}$ & $\left(h / \eta_{g}\right)_{\max }$ & $\left(h / \eta_{l}\right)_{\max }$ & $\left\langle\varepsilon_{u}\right\rangle_{n r m}$ & $\left\langle\varepsilon_{\theta}\right\rangle_{n}$ \\
\hline & 0 & 219 & 17.02 & 16.98 & 17.00 & $0.23 \%$ & 7 & 1.34 & 1.77 & 0.977 & 0.97 \\
\hline & 0.3 & 226 & 15.97 & 15.94 & 15.98 & $0.19 \%$ & 7 & 1.31 & 1.67 & 0.977 & .97 \\
\hline$R a=10^{7}$ & 0.5 & 230 & 14.65 & 14.60 & 14.64 & $0.34 \%$ & 7 & 1.29 & 1.60 & 0.979 & - \\
\hline$N=128$ & 0.6 & 225 & 13.97 & 14.03 & 13.98 & $0.43 \%$ & 8 & 1.27 & 1.56 & 0.978 & $.97 \mathrm{C}$ \\
\hline \multirow[t]{5}{*}{ Standard } & 0.8 & 652 & 12.42 & 12.39 & 12.42 & $0.24 \%$ & 8 & 1.23 & 1.51 & 0.978 & .97 \\
\hline & 1.0 & 622 & 11.38 & 11.48 & 11.38 & $0.87 \%$ & 9 & 1.20 & 1.44 & 0.977 & .9 \\
\hline & 2.0 & 321 & 12.44 & 12.35 & 12.34 & $0.45 \%$ & 9 & 1.22 & 1.54 & 0.978 & 0.9 \\
\hline & 0 & 656 & 32.18 & 32.23 & 32.20 & $0.15 \%$ & 10 & 1.52 & 2.00 & 0.974 & 098 \\
\hline & 0.3 & 472 & 30.36 & 30.39 & 30.31 & $0.10 \%$ & 10 & 1.50 & 1.88 & 0.976 & .9 \\
\hline$R a=10^{8}$ & 0.5 & 634 & 27.50 & 27.40 & 27.47 & $0.36 \%$ & 11 & 1.46 & 1.84 & 0.981 & 0.97 \\
\hline$N=256$ & 0.6 & 705 & 25.99 & 26.15 & 26.04 & $0.59 \%$ & 12 & 1.44 & 1.82 & 0.979 & $0.9^{9}$ \\
\hline \multirow[t]{5}{*}{ Standard } & 0.8 & 448 & 22.69 & 22.74 & 22.70 & $0.22 \%$ & 13 & 1.38 & 1.77 & 0.983 & 0.97 \\
\hline & 1.0 & 911 & 25.17 & 25.41 & 25.16 & $0.95 \%$ & 11 & 1.43 & 1.79 & 0.977 & $0.9^{9}$ \\
\hline & 20.0 & 168 & 15.19 & 15.30 & 15.15 & $0.70 \%$ & 74 & 1.26 & 2.17 & 1.001 & 0.9 \\
\hline & 0 & 647 & 63.98 & 63.82 & 63.95 & $0.25 \%$ & 6 & 315 & 4.53 & 0.889 & 0.91 \\
\hline & 0.3 & 647 & 60.75 & 60.82 & 60.74 & $0.12 \%$ & 6 & 3.11 & 4.21 & 0.894 & 0.90 \\
\hline$R a=10^{9}$ & 0.5 & 677 & 54.50 & 54.48 & 54.41 & $0.03 \%$ & 7 & 3.03 & 4.26 & 0.901 & 0.90 \\
\hline$N=256$ & 0.6 & 356 & 50.48 & 50.74 & 50.92 & $0.53 \%$ & 7 & 2.98 & 4.29 & 0.904 & 0.9 \\
\hline \multirow[t]{4}{*}{ Coarse } & 0.8 & 819 & 45.66 & 45.37 & 45.55 & $0.64 \%$ & 7 & 2.90 & 4.29 & 0.916 & 0.9 \\
\hline & 1.0 & 763 & 55.56 & 55.63 & 55.85 & $0.14 \%$ & 6 & 3.04 & 4.13 & 0.900 & 0.9 \\
\hline & 0 & 177 & 63.10 & 63.20 & 63.12 & $0.15 \%$ & 16 & 1.69 & 2.25 & 0.965 & 0.97 \\
\hline & 0.3 & 142 & 59.68 & 60.11 & 60.12 & $0.72 \%$ & 16 & 1.67 & 2.16 & 0.964 & 0.97 \\
\hline$R a=10^{9}$ & 0.5 & 147 & 54.72 & 54.28 & 54.80 & $0.79 \%$ & 17 & 1.63 & 2.20 & 0.970 & 0.9 \\
\hline$N=512$ & 0.6 & 163 & 50.25 & 50.63 & 50.40 & $0.76 \%$ & 19 & 1.60 & 2.19 & 0.974 & 0.9 \\
\hline \multirow[t]{2}{*}{ Standard } & 0.8 & 353 & 44.36 & 44.25 & 44.50 & $0.25 \%$ & 20 & 1.55 & 2.18 & 0.977 & 0.97 \\
\hline & 1.0 & 240 & 55.86 & 55.35 & 55.83 & $0.92 \%$ & 16 & 1.64 & 2.17 & 0.968 & 0.9 \\
\hline \multirow{4}{*}{$\begin{array}{l}R a=10^{9} \\
N=1024 \\
\text { Fine }\end{array}$} & & & & & & & & & & & \\
\hline & 0.8 & 68 & 45.11 & 44.17 & 45.52 & $2.10 \%$ & 48 & 0.81 & 1.10 & 1.008 & 0.9 \\
\hline & 0 & 32 & 127.81 & 126.72 & 128.37 & $0.85 \%$ & 24 & 1.85 & 2.62 & 0.947 & 0.96 \\
\hline & 0.3 & 32 & 118.79 & 119.81 & 119.71 & $0.86 \%$ & 26 & 1.82 & 2.56 & 0.954 & 0.96 \\
\hline$R a=10^{10}$ & 0.5 & 41 & 109.70 & 107.32 & 108.85 & $2.17 \%$ & 28 & 1.78 & 2.67 & 0.962 & 0.9 \\
\hline$N=1024$ & 0.6 & 44 & 102.59 & 102.64 & 101.34 & $0.05 \%$ & 30 & 1.75 & 2.76 & 0.973 & 0.9 \\
\hline \multirow[t]{2}{*}{ Standard } & 0.8 & 56 & 102.64 & 102.08 & 107.68 & $0.55 \%$ & 27 & 1.76 & 2.75 & 0.952 & 0.964 \\
\hline & 1.0 & 53 & 118.07 & 115.21 & 117.60 & $2.42 \%$ & 24 & 1.79 & 2.20 & 0.926 & 0.961 \\
\hline
\end{tabular}

TABLE 2. Simulation parameters and global quantities for parameter and grid-convergence study. The number of grid points $N$ is the same in all the three directions. Here $\tilde{\tau}_{\text {avg }}=\tau_{\text {avg }} U / H$ is the averaging time period; $N u \equiv(H / \Delta)|\mathrm{d} \bar{\theta} / \mathrm{d} z|_{w}$ is the Nusselt number, where $|\mathrm{d} \bar{\theta} / \mathrm{d} z|_{w}$ is the absolute wall temperature gradient, averaged over time, $x y$-plane and both walls; $N u_{h}$ is the counterpart of $N u$, averaged over the second half of $\tilde{\tau}_{\text {avg }} ; N u_{b o t} \equiv(H / \Delta)|\mathrm{d} \bar{\theta} / \mathrm{d} z|_{z=0}$ is the Nusselt number at the bottom wall averaged over $\tilde{\tau}_{\text {avg }} ;$ Diff $\tau_{\text {avg }} \equiv\left(N u-N u_{h}\right) / N u ; N_{B L}$ is the number of grid points within the thermal boundary layer located by the outer peak of root mean square (r.m.s.) of temperature fluctuation; $h=\max \left(\Delta_{x}, \Delta_{y}, \Delta_{z}\right)$ is the maximum local grid size, and $\eta_{l}(z) \equiv\left(v^{3} / \overline{\varepsilon_{u}}\right)^{1 / 4}$ and $\eta_{g} \equiv\left(v^{3} /\left\langle\varepsilon_{u}\right\rangle\right)^{1 / 4}$ are the local and global Kolmogorov length scales; $\left\langle\varepsilon_{u}\right\rangle_{n r m}=\left\langle\varepsilon_{u}\right\rangle \operatorname{Pr}^{2} H^{4} /\left[v^{3}(N u-1) R a\right]$ and $\left\langle\varepsilon_{\theta}\right\rangle_{n r m}=\left\langle\varepsilon_{\theta}\right\rangle H^{2} /\left(\kappa \Delta^{2} N u\right)$ measure the global balance between the dissipation rates and Nusselt number, a measure of resolution sufficiency (Calzavarini et al. 2005; Stevens, Verzicco \& Lohse 2010). For a perfect resolution, the criterion $\left\langle\varepsilon_{u}\right\rangle_{n r m}=\left\langle\varepsilon_{\theta}\right\rangle_{n r m}=1$ must be nearly satisfied, regardless of the scheme used to compute $\varepsilon_{u}$ and $\varepsilon_{\theta}$. See the text for discussion. 


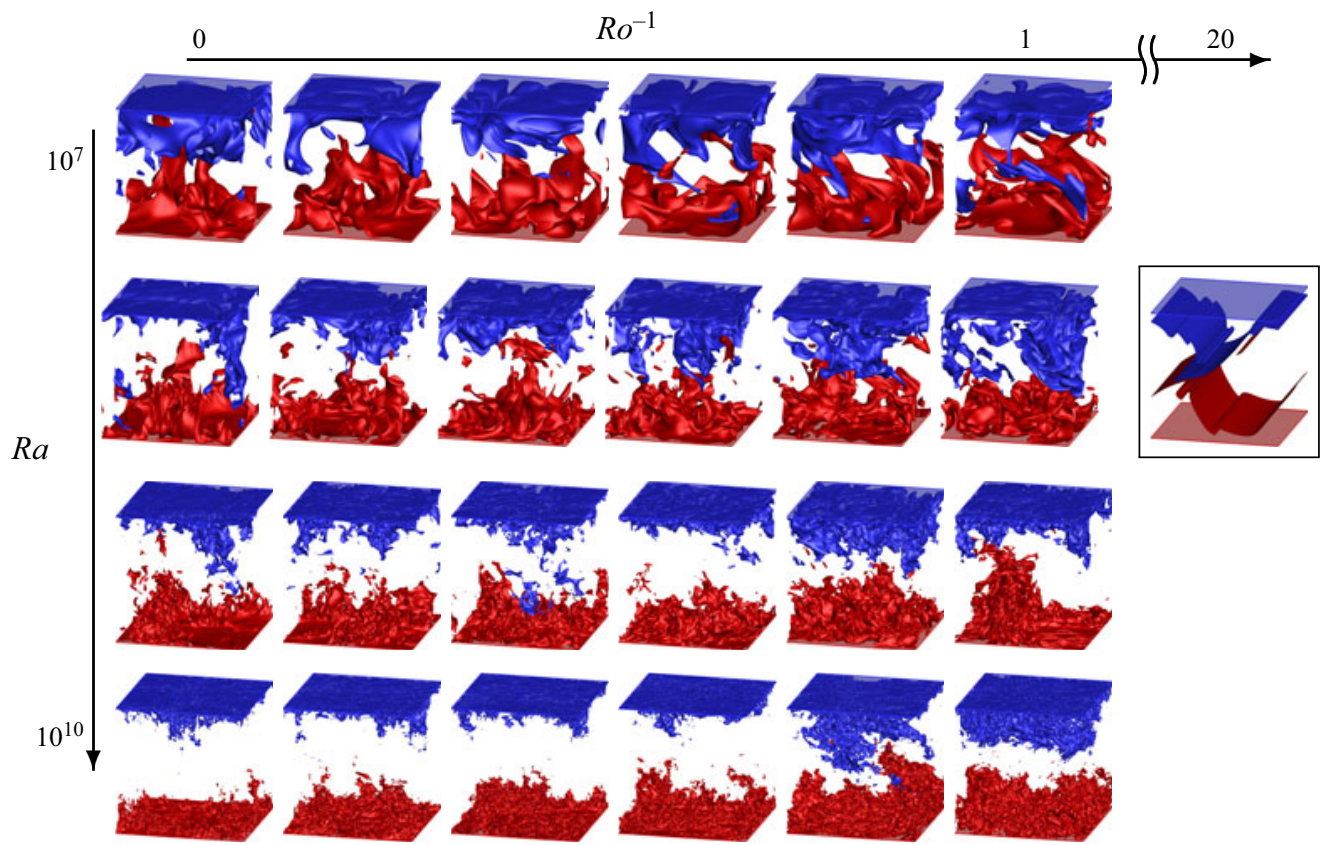

FIGURE 3. Flow visualisation of the simulation cases. The unframed cases correspond to four Rayleigh numbers $R a=\left(10^{7}, 10^{8}, 10^{9}, 10^{10}\right)$, from the first to the last row, respectively, and six inverse Rossby numbers $R o^{-1}=(0,0.3,0.5,0.6,0.8,1.0)$, from the first to the last column, respectively. The special framed case is at $R a=10^{8}, R o^{-1}=20.0$. Isosurface of $\theta=-\Delta / 10$ (blue) and $\theta=+\Delta / 10$ (red).

(36), (42) and (43)) to resolve the Kolmogorov scale both in the bulk and within the boundary layers. In table 2 , we call these resolutions standard, as we show here that they are suitable for well-resolved DNS. We also performed calculations at coarser and finer resolutions, and we call them coarse and fine, respectively. In total, four values of $R a$ are simulated, ranging from $10^{7}$ to $10^{10}$, and at each $R a, R o^{-1}$ is varied from zero (no Coriolis force) to unity (large Coriolis force). We performed two additional cases: one at $R o^{-1}=2.0$ for $R a=10^{7}$, and the other one at $R o^{-1}=20$ for $R a=10^{8}$, where the Coriolis force is much larger than inertia. We perform an extensive parameter and grid-convergence study (table 2), following the prescriptions by Stevens et al. (2010). For simulation convergence, each case is run for approximately 100 turnover times $H / U$ to discard initial transients, and data is averaged over an additional $\tau_{\text {avg }} \gtrsim 150 \mathrm{H} / \mathrm{U}$ for statistical convergence. Statistical convergence is evaluated based on the Nusselt number $N u \equiv(H / \Delta)|\mathrm{d} \bar{\theta} / \mathrm{d} z|_{w}$, where $|\mathrm{d} \bar{\theta} / \mathrm{d} z|_{w}=\left(|\mathrm{d} \bar{\theta} / \mathrm{d} z|_{z=0}+|\mathrm{d} \bar{\theta} / \mathrm{d} z|_{z=H}\right) / 2$ is the absolute wall temperature gradient, averaged over time, $x y$-plane and both walls. We call a simulation statistically converged, once the difference between $N u$ obtained from averaging over $\tilde{\tau}_{\text {avg }}$ and its counterpart $N u_{h}$ obtained from averaging over the second half of $\tilde{\tau}_{\text {avg }}$ is less than $1 \%$ (see Diff $\tau_{\tau_{a v g}}$ in table 2). We can also see the statistical convergence in the less than $1 \%$ difference between $N u$ and its counterpart at the bottom wall $N u_{\text {bot }}$. Another way for statistical convergence is when the difference between top and bottom wall Nusselt numbers $D_{\text {iff }} f_{N u}=\left|N u_{\text {top }}-N u_{\text {bot }}\right| / N u$ is negligibly small (Kunnen et al. 2016). For all cases up to $R a=10^{9}$, Diff $_{N u}$ is less than $2 \%$. 
We perform such an extensive statistical convergence study up to $R a=10^{9}$, where the resolution requirement (maximum $N=512$ ) is affordable to run the calculations for at least $250 H / U$. The cases at $R a=10^{10}$ require $N=1024$ for well-resolved simulation, and are substantially expensive. For example, running each well-resolved case at $R a=$ $10^{9}(N=512)$ for $250 H / U$, demands approximately 0.05 million central processing unit (CPU) core hours, whereas running each well-resolved case at $R a=10^{10}(N=1024)$ for $250 \mathrm{H} / \mathrm{U}$, demands approximately 2.0 million CPU core hours, 40 times more expensive than $R a=10^{9}$. Given the expensive cost at $R a=10^{10}$, each case is simulated for at least $60 \mathrm{H} / \mathrm{U}$ and statistical averaging is performed over $\tau_{\text {avg }} \gtrsim 30 \mathrm{H} / \mathrm{U}$. Running these cases to full statistical convergence does not add to the conclusions that we draw by studying the cases up to $R a=10^{9}$. Our primary aim by reporting $R a=10^{10}$ is to demonstrate some evidence of boundary layer transition to turbulent, owing to the favourable role of the Coriolis force.

Grid convergence was evaluated based on three criteria. (i) Resolving the local Kolmogorov scale $\eta_{l}(z) \equiv\left(v^{3} / \overline{\varepsilon_{u}}\right)^{1 / 4}$, where $\varepsilon_{u}=v\left(\partial u_{i} / \partial x_{j}\right)^{2}$ is the kinetic energy dissipation rate. (ii) Satisfying the global exact relations $\left\langle\varepsilon_{u}\right\rangle=v^{3}(\mathrm{Nu}-1) \mathrm{RaPr}^{-2} / \mathrm{H}^{4}$ and $\left\langle\varepsilon_{\theta}\right\rangle=\kappa \Delta^{2} N u / H^{2}$, where $\varepsilon_{\theta}=\kappa\left(\partial \theta / \partial x_{j}\right)^{2}$ is the thermal energy dissipation rate. (iii) Comparison of parameters of interest between sequentially finer grid resolutions.

Criterion (i) was initially prescribed by Grötzbach (1983), suggesting $h \leq \pi \eta_{g}$, where $h=\left(\Delta_{x} \Delta_{y} \Delta_{z}\right)^{1 / 3}$ is the grid size and $\eta_{g} \equiv\left(v^{3} /\left\langle\varepsilon_{u}\right\rangle\right)^{1 / 4}$ is the global Kolmogorov scale, based on the volume and time-averaged dissipation rate. Grötzbach (1983) ignored the anisotropy in the grid (by using the geometric mean for $h$ ) and heterogeneity in the dissipation rate (by integrating $\varepsilon_{u}$ over the domain and time). Stevens et al. (2010) showed that for well-resolved simulations, the anisotropic grid and flow heterogeneity must be taken into account. Therefore, following Stevens et al. (2010) we chose $h=$ $\max \left(\Delta_{x}, \Delta_{y}, \Delta_{z}\right)$, and calculated the local Kolmogorov scale $\eta_{l}$ in each height based on $\overline{\varepsilon_{u}}$. Also we calculated the global Kolmogorov scale $\eta_{g}$, and in table 2 we compare the maximum ratios $\left(h / \eta_{g}\right)_{\max }$ and $\left(h / \eta_{l}\right)_{\max }$. As observed, resolving $\eta_{l}$ demands finer resolution than resolving $\eta_{g}$. Results of Stevens et al. (2010) and our results in table 2 show that the criterion $\left(h / \eta_{g}\right)_{\max } \leq \pi$ is not sufficient for well-resolved simulation. For instance, the simulations at $R a=10^{9}$ with $N^{3}=256^{3}$, satisfy $\left(h / \eta_{g}\right)_{\max } \leq \pi$ but not $\left(h / \eta_{l}\right)_{\max } \leq \pi$, and they poorly satisfy the global exact relations for criterion (ii) $\left(\left\langle\varepsilon_{u}\right\rangle_{n r m} \simeq\left\langle\varepsilon_{\theta}\right\rangle_{n r m} \simeq 0.90\right)$. For a perfect resolution, the criterion $\left\langle\varepsilon_{u}\right\rangle_{n r m}=\left\langle\varepsilon_{\theta}\right\rangle_{n r m}=1.0$ must be nearly satisfied, even if $\varepsilon_{u}$ and $\varepsilon_{\theta}$ are calculated with a different scheme than the code discretisation scheme. Here, we use a fourth-order kinetic energy conservative and a third-order scalar variance non-conservative code. We compute $\varepsilon_{u}$ and $\varepsilon_{\theta}$ using a second-order central differencing scheme. We obtain $\left\langle\varepsilon_{u}\right\rangle_{n r m} \simeq\left\langle\varepsilon_{\theta}\right\rangle_{n r m} \simeq 0.97$, for all the standard resolution cases, similar to Stevens et al. (2010). These standard resolution cases also satisfy criterion (i), i.e. $\left(h / \eta_{l}\right)_{\max } \leq \pi$.

In figure 4 , we show the adequacy of standard resolution based on criterion (iii). We consider $R a=10^{9}$ and $R o^{-1}=0.8$, comparing $\overline{\varepsilon_{u}}$ and $\overline{\varepsilon_{\theta}}$ between three grid resolutions: $N=256$ (coarse), 512 (standard) and 1024 (fine). At the coarse resolution $(N=256$ ), these quantities are slightly lower than the ones at the standard and fine resolutions (consider the insets in figure 4). However, the difference between the standard and fine resolutions is negligible. According to Stevens et al. (2010), in the under-resolved simulations the gradients are smeared out, and $\varepsilon_{u}$ and $\varepsilon_{\theta}$ are underestimated. The results that we present in the rest of this paper (figure 3), correspond to the well-resolved standard resolution cases (table 2). 

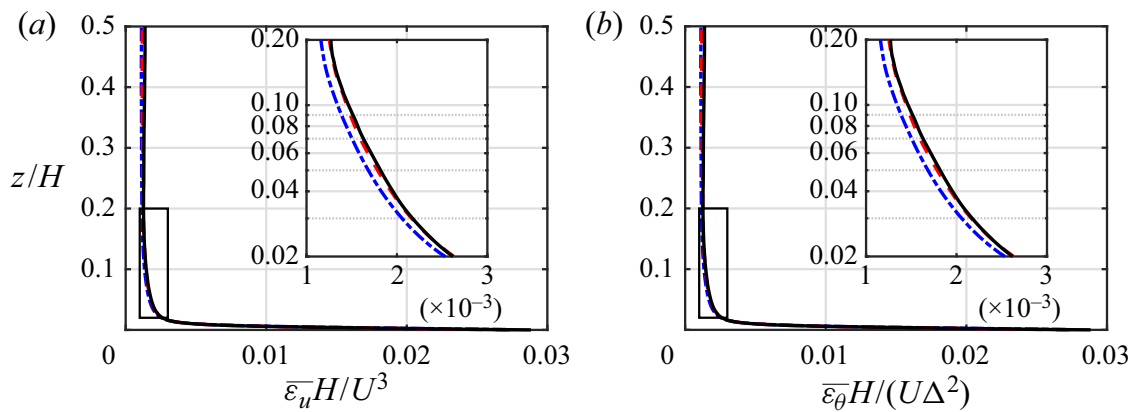

FIGURE 4. Comparison of (a) kinetic energy dissipation rate $\overline{\varepsilon_{u}}$ and $(b)$ thermal energy dissipation rate $\overline{\varepsilon_{\theta}}$, averaged over $x y$-plane and time, at $R a=10^{9}$ and $R o^{-1}=0.8$ between three grid resolutions $N$ : coarse 256 (dashed-dotted blue line), standard 512 (dashed red line) and fine 1024 (solid black line). The insets show the framed areas.

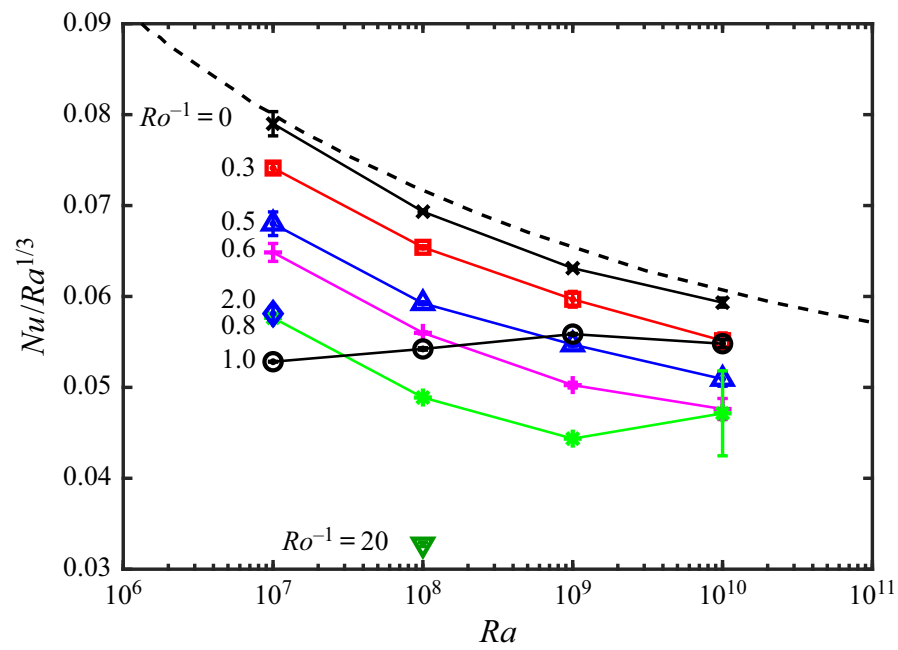

FigURE 5. Here $N u / R a^{1 / 3}$ for all the standard-resolution cases listed in table 2. Here $N u=$ $(H / \Delta)|\mathrm{d} \bar{\theta} / \mathrm{d} z|_{w}$, where $|\mathrm{d} \bar{\theta} / \mathrm{d} z|_{w}=\left(|\mathrm{d} \bar{\theta} / \mathrm{d} z|_{z=0}+|\mathrm{d} \bar{\theta} / \mathrm{d} z|_{z=H}\right) / 2 ; R o^{-1}=0(\times$, black $), 0.3(\square$, red), $0.5(\triangle$, blue), 0.6 ( + , magenta), $0.8(*$, green), 1.0 (०, black), $2.0(\diamond$, blue) and $20.0(\nabla$, olive green). Grossmann \& Lohse (2000) theory with the parameters as fixed in Stevens et al. (2013b) (dashed black line). If the error bar $\left|N u_{t o p}-N u_{b o t}\right| / R a^{1 / 3}$ is not visible, it is smaller than the symbol size.

\section{Results}

\subsection{Effect of the Coriolis force on heat and momentum fluxes}

The resulting Nusselt number $N u=(H / \Delta)|\mathrm{d} \bar{\theta} / \mathrm{d} z|_{w}$ for all cases is compiled in figure 5 . When $R o^{-1}=0(\times$, no Coriolis force), $\mathrm{Nu}$ is close to the Grossmann \& Lohse (2000) theory (dashed black line), as expected. At each $R a$, as $R o^{-1}$ increases (i.e. Coriolis force increases), $\mathrm{Nu}$ decreases until it reaches a minimum at an optimal $R o_{o p t}^{-1}$; beyond $R o_{\text {opt }}^{-1}, N u$ increases. This is better shown in figure 6(a), plotting $N u$ versus $R o^{-1}$, at each $R a$. Additionally, in figure $6(b)$ we plot the skin-friction coefficient $C_{f}=2 v|\mathrm{~d} \bar{u} / \mathrm{d} z|_{w} / U^{2}$ versus $R o^{-1}$, at each $R a$. Here $|\mathrm{d} \bar{u} / \mathrm{d} z|_{w}$ is the modulus of the wall velocity gradient, 

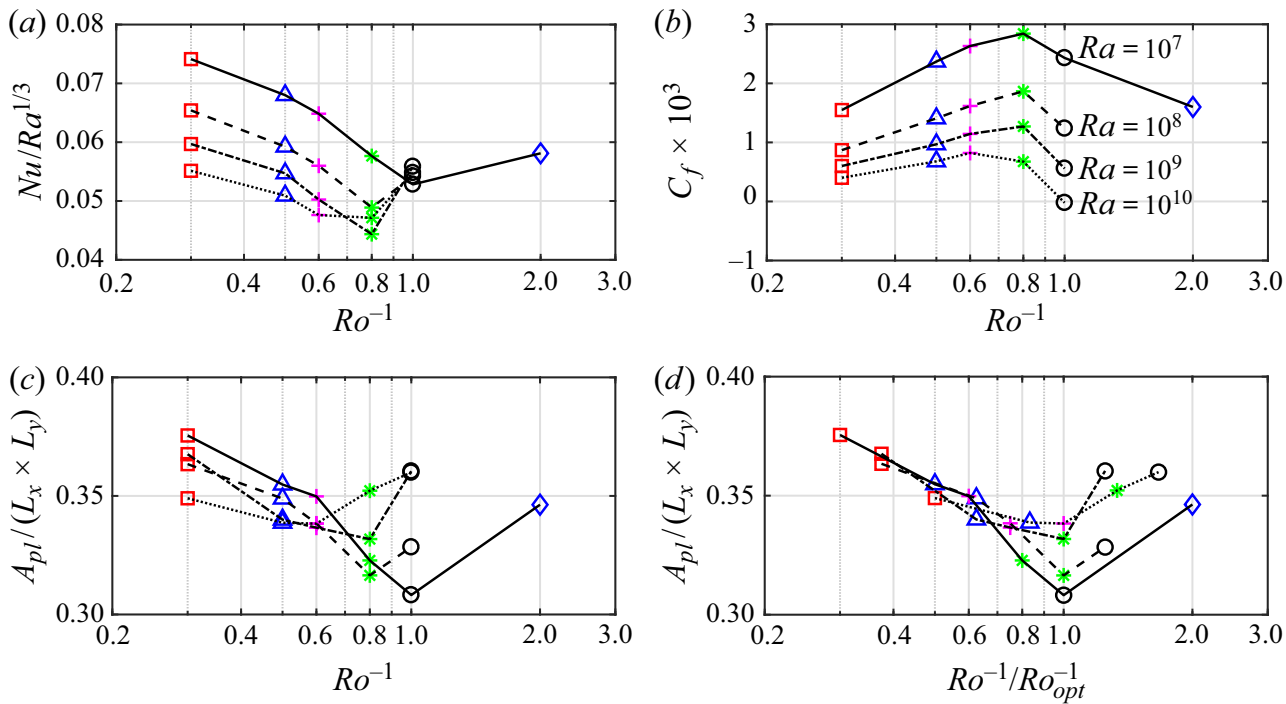

Figure 6. Variation of (a) $N u / R a^{1 / 3}$, (b) $C_{f}$ and $(c, d) A_{p l}$ cold plume coverage at the edge of the bottom wall thermal boundary layer, versus $R o^{-1}$ at different $R a$. Panel $(d)$ is the same as panel (c) except $R o^{-1}$ is scaled by $R o_{o p t}^{-1}$, the value of $R o^{-1}$ at minimum $A_{p l}$. Here $C_{f}=$ $2 v|\mathrm{~d} \bar{u} / \mathrm{d} z|_{w} / U^{2}$, where $|\mathrm{d} \bar{u} / \mathrm{d} z|_{w}=\left(|\mathrm{d} \bar{u} / \mathrm{d} z|_{z=0}+|\mathrm{d} \bar{u} / \mathrm{d} z|_{z=H}\right) / 2 ; R a=10^{7}$ (solid black line), $10^{8}$ (dashed black line), $10^{9}$ (dashed-dotted black line) and $10^{10}$ (dotted black line). Each symbol corresponds to one $R o^{-1}$ consistent with figure 5. Note that in panel $(b)$ at $R a=10^{10}$ and $R o^{-1}=$ 1.0 ( $\circ$, black), $C_{f} \simeq 2 \times 10^{-5}$ ( $\simeq 0$ on the given linear scale for $C_{f}$ ), where the flow is at the onset of reversal.

averaged over time, $x y$-plane and both walls. Similar to $N u$, at each $R a$ there is an optimal $R o_{o p t}^{-1}$ at which $C_{f}$ becomes maximal. Comparing figure $6(a)$ with $6(b)$, the values of $R o_{o p t}^{-1}$ for the minimum in $\mathrm{Nu}$ and the maximum in $C_{f}$ are close to each other, hence minimal heat flux coincides with maximal skin friction. Here $R o_{o p t}^{-1}$ depends on $R a$, decreasing from approximately 1.0 at $R a=10^{7}$, to approximately 0.6 at $R a=10^{10}$.

To explain the underlying mechanism for the behaviour seen in $N u$ and $C_{f}$ versus $R o^{-1}$, we study the flow at different values of $R o^{-1}$ (figure 7). We focus on $R a=10^{8}$, but our conclusions can be generalised to other values of $R a$. In figure $7(e-h)$, we show the instantaneous spanwise averaged temperature field $\theta^{y} / \Delta$, overlaid by the instantaneous spanwise averaged velocity vector $\left(u^{y} / U, w^{y} / U\right)$. Comparing the flow at an $R o^{-1}$ smaller than $R o_{o p t}^{-1}$ (figure $7 a, e$ ), equal to $R o_{o p t}^{-1}$ (figure $7 b, f$ ), larger than $R o_{o p t}^{-1}$ (figure $7 c, g$ ) and much larger than $R o_{o p t}^{-1}$ (figure $7 d, h$ ), we see that up to $R o_{o p t}^{-1}$ the hot fluid is mainly driven in the positive $x$-direction and the cold fluid is mainly driven in the negative $x$-direction. This is better seen in the mean velocity profiles (solid red line, solid green line) in figure $8(a)$. In fact, up to $R o_{o p t}^{-1}=0.8$ an antisymmetric bidirectional wind is formed, that drives the flow near the top and bottom walls in the opposite directions. At $R o_{o p t}^{-1}=0.8$ (figure $7 b, f$ ), the wind gains the maximum momentum, hence maximal $C_{f}$, and the velocity profile in wall units (solid green line in figure $8 b$ ) moves closer to the Prandtl-von Kármán (logarithmic) profile. Beyond $R o_{o p t}^{-1}$ (figure $7 c, g$ ), the wind is weakened and becomes asymmetric, hence $C_{f}$ decreases (figure $6 b$ ). In appendix A, we conclude that the asymmetric flow is a persistent statistical state. Our conclusion is based on simulating the case in figure $7(c, g)$ with two different initial conditions and running each calculation for approximately 1200 


$\begin{array}{lll}0.3 & R o^{-1} \\ 0.1 & & 1.0\end{array}$

(a)

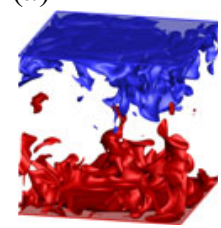

$(f)$

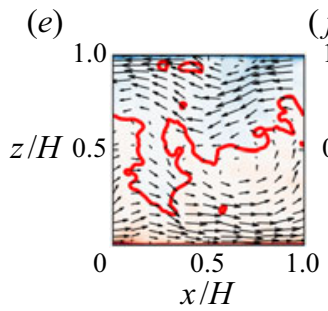

(b)

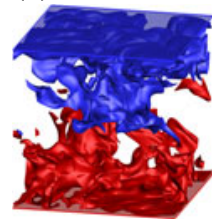

(c)

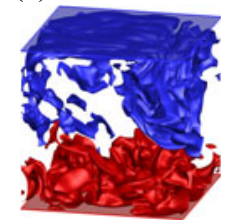

( $g)$

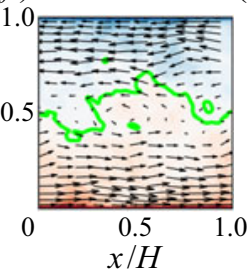

(g)

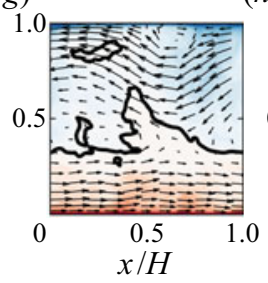

(d)

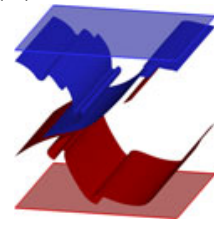

(h)

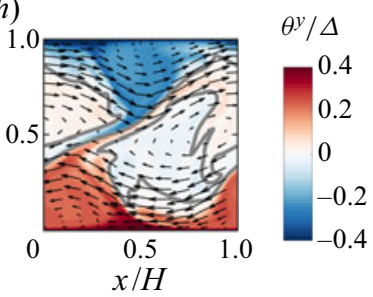

Figure 7. Visualisation of the flow at $R a=10^{8}$ and $R o^{-1}=0.3(a, e), R_{o}^{-1}=0.8(b, f)$, $R o^{-1}=1.0(c, g)$ and $R o^{-1}=20.0(d, g)$. $(a-d)$ Isosurface of $\theta=-\Delta / 10$ (blue) and $\theta=$ $+\Delta / 10$ (red). $(e-h)$ Instantaneous $y$-averaged velocity vector $\left(u^{y} / U, w^{y} / U\right)$, overlaid by the instantaneous $y$-averaged temperature field $\left(\theta^{y} / \Delta\right)$; the thick line in the domain locates $\theta^{y}=0$.

(a)

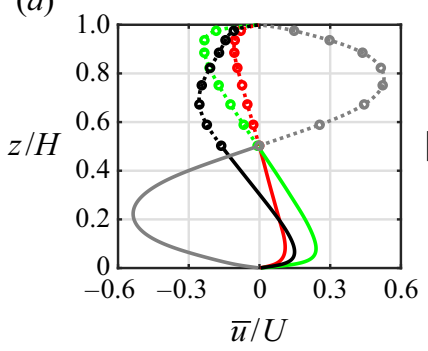

(b)

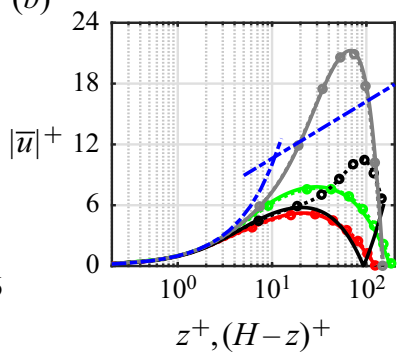

(c)

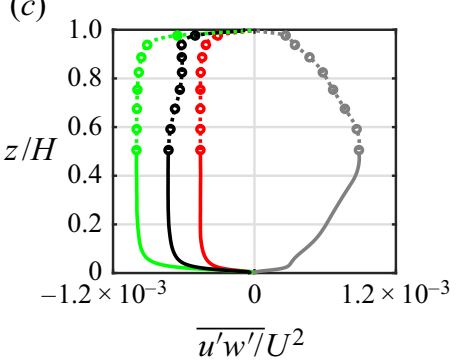

FIGURE 8. Flow statistics for the cases shown in figure 7 at $R a=10^{8}$ and $R o^{-1}=0.3$ (solid red line), 0.8 (solid green line), 1.0 (solid black line) and 20.0 (solid grey line). Mean velocity profiles $(a, b)$, scaled by $U, H(a)$ and $u_{\tau}, v(b)$, and Reynolds shear stress profiles $(c)$ scaled by $U, H$. The line style is solid for $0 \leq z \leq H / 2$ and dot-circle for $H / 2 \leq z \leq H$. Law of the wall (dashed-dotted blue line) in the viscous sublayer $\bar{u}^{+}=z^{+}$, and log layer (Prandtl-von Kármán profile) $\bar{u}^{+}=(1 / 0.41) \ln \left(z^{+}\right)+5.2$ (Yaglom 1979).

turnover times. In the extreme case of $\mathrm{Ro}^{-1}=20$ (figure $7 d, h$ ), turbulence is completely suppressed, the flow is two-dimensional and laminar. At this stage the mean velocity profile is reversed (figure $8 a$ ). The flow reversal occurs at a lower $R o^{-1}$ as $R a$ increases, such that at $R a=10^{10}$ it occurs at $R o^{-1} \simeq 1.0$. In figure 6(b), $C_{f} \simeq 0$ at $R a=10^{10}$ and $R o^{-1}=1.0$, implying the onset of flow reversal.

The bidirectional wind also appears in centrifugal convection with free-slip hot and cold boundaries (von Hardenberg et al. 2015; Novi et al. 2019). In appendix B, we compare this system (CC_slip in table 1) with our system (CC_wall in table 1). We see several differences due to different boundary conditions. In CC_slip, the bidirectional wind never 
breaks down, but in CC_wall it breaks down. As a result, in CC_slip there is no optimal $R o_{o p t}^{-1}$, but in CC_wall there is an $R o_{o p t}^{-1}$. Also, in CC_slip the bidirectional wind can have a cyclonic or anticyclonic mean vorticity (von Hardenberg et al. 2015), but in CC_wall the bidirectional wind is always anticyclonic (appendix $\mathrm{A}$ ).

The different flow regimes caused by changing $R^{-1}$ (Coriolis force) also explains the variations in $N u$ (figure $6 a$ ). The variations in $N u$, i.e. heat transfer, is related to the vertical fluid motion between the end walls. The vertical fluid motion is qualitatively observed by the isoline of $\theta^{y}=0$ in figure $7(e-h)$. At small $R o^{-1}$, small Coriolis force (figure 7e), the isoline of $\theta^{y}=0$ (solid red line) highlights the upwelling and downwelling thermal plumes, as seen in Rayleigh-Bénard convection (Ahlers, Grossmann \& Lohse 2009), hence, $N u$ is closer to the Grossmann \& Lohse (2000) theory (figure 5). At $R o_{o p t}^{-1}$ (figure $7 f$ ), the bidirectional wind inhibits the heat exchange between the end walls by suppressing the vertical fluid motion. The isoline of $\theta^{y}=0$ (solid green line) mainly stays at the midheight of the domain, indicating that the hot and cold fluids are mainly locked to the lower and upper halves of the domain, respectively; therefore, wall temperature gradients (i.e. $\mathrm{Nu}$ ) are minimum. At $R o^{-1}>R o_{o p t}^{-1}$ (figure $7 g$ ), the bidirectional wind is weakened and vertical fluid motion starts to form; thus, $N u$ starts to increase. To quantify the exchange of hot and cold fluids between the end walls (i.e. heat exchange), following Chong et al. (2017) in figure 6(c,d) we plot the area ratio $A_{p l} /\left(L_{x} \times L_{y}\right)$ covered by the cold fluid at the edge of the bottom hot thermal boundary layer $\delta_{\theta}$ (where $\sqrt{\overline{\theta^{\prime 2}}}$ is maximum). We sum over the areas at which $\left(\theta-\theta^{x y}\right) \leq-\left.0.5 \sqrt{\overline{\theta^{\prime 2}}}\right|_{\text {ref }}$, where $\left.\sqrt{\overline{\theta^{\prime 2}}}\right|_{\text {ref }}$ is a unified threshold corresponding to $R o^{-1}=0$ at $\delta_{\theta}$. Comparing figure 6(a) with $6(c)$ shows that the variations in $N u$ and $A_{p l}$ are consistent with each other. At $R o_{o p t}^{-1}$, owing to the bidirectional wind, the cold fluid coverage at the edge of the bottom thermal boundary layer reaches minimum $\left(A_{p l}\right.$ is minimum). Consequently, the heat exchange between the end walls reaches its minimum ( $N u$ is minimal).

Our study shows that the interaction between the Coriolis force and buoyancy force creates different flow regimes. When the buoyancy force is stronger than the Coriolis force $\left(R o^{-1} \ll R o_{o p t}^{-1}\right)$, the flow is similar to Rayleigh-Bénard convection. Vice versa, when the Coriolis force is stronger than buoyancy $\left(R o^{-1} \gg R o_{o p t}^{-1}\right)$, the flow becomes laminar. At an intermediate force balance $\left(R o_{o p t}^{-1}\right)$, optimal transport occurs with maximal $C_{f}$ and minimal $N u$. Similar flow regimes are reported by Jiang et al. (2020) (see their figure 2). They perform DNS of a full cylindrical shell with finite shell thickness $(H / R \simeq 0.5)$. Their variation in $N u$ versus $R o^{-1}$ is similar to figure 6(a); a minimal $N u$ occurs at an optimal $R o^{-1}$.

Optimal transport also occurs in rotating Rayleigh-Bénard convection (see the review by Stevens, Clercx \& Lohse (2013a)), but only in rot_RB_wall and rot_RB_cyl with hot and cold walls (table 1). For rot_RB_wall see Julien et al. (1996b), King et al. (2009, 2012), Pieri et al. (2016) and Chong et al. (2017); and for rot_RB_cyl see Kunnen et al. (2008), Liu \& Ecke (2009), Stevens et al. (2009, 2011), Zhong et al. (2009), Zhong \& Ahlers (2010), Kunnen et al. (2011), Weiss \& Ahlers (2011a,b), Ecke \& Niemela (2014), Horn \& Shishkina (2014) and Zhang et al. (2020). In these systems, optimal transport roughly coincides with the transition between vertically coherent columns (Taylor columns) and vertically spinning plumes (Julien et al. 2012; Stellmach et al. 2014; Cheng et al. 2015; Kunnen et al. 2016). These structures are emanated from the Ekman layers at the walls. As a result, optimal vertical transport (maximal $\mathrm{Nu}$ ) occurs, as opposed to optimal horizontal transport (minimal $\mathrm{Nu}$ ) in centrifugal convection. This difference is due to different axis 
of rotation between rotating Rayleigh-Bénard convection and centrifugal convection. Novi et al. (2019) changed the angle between the buoyancy force and axis of rotation from zero (rotating Rayleigh-Bénard convection) to $90^{\circ}$ (centrifugal convection). They observed that the flow structures evolve from columnar vortices to bidirectional wind. In rot_RB_slip with free-slip hot and cold boundaries (table 1), no Ekman layer forms. Therefore, no optimal transport occurs (Julien et al. 1996b; Schmitz \& Tilgner 2009, 2010). Optimal transport occurs in other systems where buoyancy (as a destabilising mechanism) interacts with a stabilising mechanism (Chong et al. 2017). Stabilising mechanisms are confinement in confined Rayleigh-Bénard convection (Chong et al. 2015), salinity in double diffusive convection (Yang, Verzicco \& Lohse 2016) or Lorentz force in magnetoconvection (Lim et al. 2019). In these flows, the interplay between the stabilising and destabilising mechanisms forms coherent structures (e.g. Taylor columns). These structures manipulate the flow towards the optimal transport (Chong et al. 2017).

\subsection{Formation of bidirectional wind}

The bidirectional wind is formed once we rotate the system, hence a combination of the Coriolis force and buoyancy force generates the wind. To investigate the underlying mechanism, we study the momentum equations $((2.1 b)-(2.1 d))$ averaged over time and the $x y$-plane,

$$
\frac{\mathrm{d} \overline{\tilde{u}^{\prime} \tilde{w}^{\prime}}}{\mathrm{d} \tilde{z}}=\left(\frac{R a}{P r}\right)^{-1 / 2} \frac{\mathrm{d}^{2} \overline{\tilde{u}}}{\mathrm{~d} \tilde{z}^{2}}, \quad \frac{\mathrm{d} \overline{\tilde{w}^{\prime} \tilde{w}^{\prime}}}{\mathrm{d} \tilde{z}}=-\frac{\mathrm{d} \overline{\tilde{p}}}{\mathrm{~d} \tilde{z}}+R o^{-1} \overline{\tilde{u}}+\overline{\tilde{\theta}},
$$

where $(3.1 a, b)$ are the averaged $u$ - and $w$-momentum equations, respectively. If we integrate $(3.1 a)$ from $\tilde{z}=0$ to an arbitrary $\tilde{z}$, we obtain $\overline{\tilde{u}^{\prime} \tilde{w}^{\prime}}=(\operatorname{Ra} / P r)^{-1 / 2} \mathrm{~d} \overline{\tilde{u}} / \mathrm{d} \tilde{z}-$ $(R a / P r)^{-1 / 2} \mathrm{~d} \overline{\tilde{u}} /\left.\mathrm{d} \tilde{z}\right|_{\tilde{z}=0}$. We can draw two conclusions from this equation. First, considering this equation at $\tilde{z}=1$ (top wall), we obtain $\mathrm{d} \overline{\tilde{u}} /\left.\mathrm{d} \tilde{z}\right|_{\tilde{z}=0}=\mathrm{d} \overline{\tilde{u}} /\left.\mathrm{d} \tilde{z}\right|_{\tilde{z}=1}$, hence the wall shear stresses at the top and bottom walls have equal magnitudes but opposite signs $\left(\bar{\tau}_{w_{\text {top }}}=\right.$ $\left.-\rho_{o} v \mathrm{~d} \bar{u} /\left.\mathrm{d} z\right|_{z=H}, \bar{\tau}_{w_{b o t}}=\rho_{o} v \mathrm{~d} \bar{u} /\left.\mathrm{d} z\right|_{z=0}\right)$. Second, if we further integrate this equation from $\tilde{z}=0$ to 1 , we obtain $C_{f_{\text {top }}}=C_{f_{\text {bot }}}=2\left|\int_{0}^{1} \overline{\tilde{u}^{\prime} \tilde{w}^{\prime}} \mathrm{d} \tilde{z}\right|$, hence the wind momentum is adjusted by the integral of Reynolds shear stress. These two conclusions must be valid for all values of $R o^{-1}(3.1 a, b)$. The first conclusion can be confirmed from figure $8(b)$, comparing the mean velocity profiles near the bottom wall (solid line) and top wall (dot-circled line). The profiles within a distance of 20 wall units from the end walls are identical to each other, even at $R o^{-1}=1$ (solid black line), where the antisymmetric wind is broken. From the second conclusion, the Coriolis and buoyancy forces must generate the wind through $\overline{u^{\prime} w^{\prime}}$. Inspecting $(3.1 b)$ shows that the Coriolis force $\left(R o^{-1} \bar{u}\right)$ and buoyancy force $(\bar{\theta})$ can modify the wall-normal Reynolds stress $\overline{w^{\prime} w^{\prime}}$, which in turn modifies $\overline{u^{\prime} w^{\prime}}$. At $R o_{o p t}^{-1}=0.8$ (solid green line), where the wind momentum reaches maximum, $\overline{u^{\prime} w^{\prime}}$ is maximum at all heights (figure $8 c$ ). At $R o^{-1}=20$ (solid grey line), the flow is laminar and $\overline{u^{\prime} w^{\prime}}$ is due to the flow unsteadiness $\left(u^{\prime}=(u-\bar{u})\right.$ is the unsteady component). Nevertheless, $\left|\int_{0}^{H} \overline{u^{\prime} w^{\prime}} \mathrm{d} z\right|$ at $R o^{-1}=20$ is still smaller than its counterpart at $R o_{o p t}^{-1}=0.8$.

\subsection{Flow behaviour beyond the optimal Coriolis force}

As discussed in $\$ 3.1$, beyond $R o_{o p t}^{-1}$ (figure $7 c, g$ ), the strong bidirectional wind is weakened and loses its antisymmetric nature. We visualise this case $\left(R a=10^{8}\right.$ and $R o^{-1}=1.0>$ $R o_{o p t}^{-1}=0.8$ ) in figure 9 for a period of approximately $4 H / U$ (indicated in the $x y$-plane averaged wall shear-stress $\tau_{w}^{x y}$ history). We see that wind breaking is coincident with 
(a)

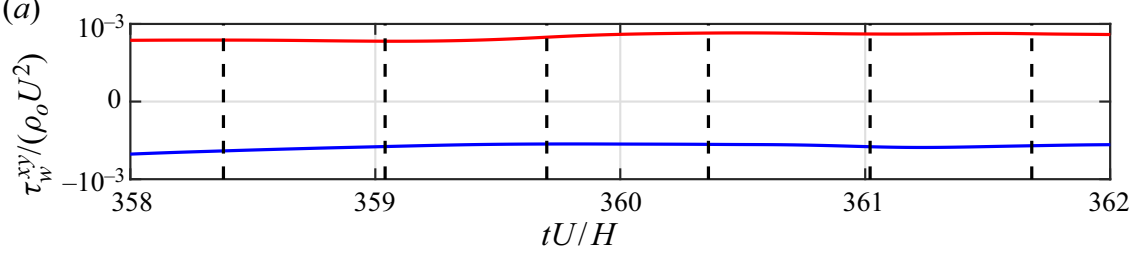

(b)

(c)

(d)

(e)

$(f)$

( $g)$

$\omega_{y}^{y} H / U$

$z / H 0.5$
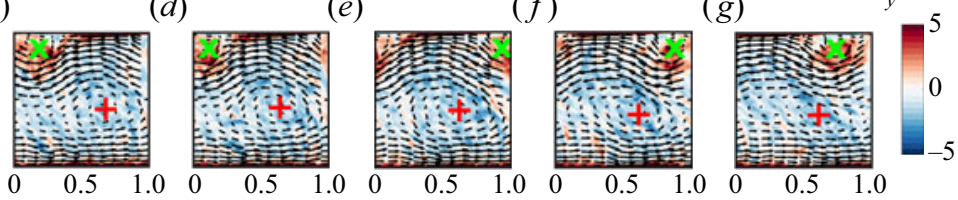

(h)

(i)

$(j)$

(k)
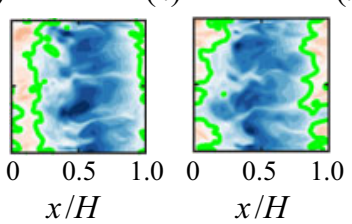

(l)

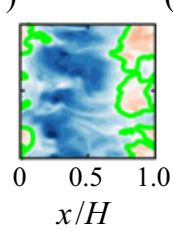

(m)

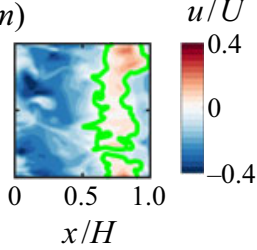

FIGURE 9. Visualisation of cyclones $\left(\times\right.$, green, $\left.\omega_{y}^{y}>0\right)$ and anticyclones $\left(+\right.$, red, $\left.\omega_{y}^{y}<0\right)$ at different instantaneous times for $R a=10^{8}, R o^{-1}=1>R o_{\text {opt }}^{-1}\left(R o_{\text {opt }}^{-1}=0.8\right)$, where $\omega_{y}^{y}=$ $\left(\partial u^{y} / \partial z-\partial w^{y} / \partial x\right)$ is the spanwise-averaged spanwise vorticity. The visualisation times from panel $(b)$ to panel $(g)$ are indicated by vertical dashed lines in the $\tau_{w}^{x y}$ history, from left to right, where $\tau_{w_{b o t}}^{x y}=-\rho_{o} v \mathrm{~d} u^{x y} /\left.\mathrm{d} z\right|_{z=0}$ (solid red line) and $\tau_{w_{\text {top }}}^{x y}=-\rho_{o} v \mathrm{~d} u^{x y} /\left.\mathrm{d} z\right|_{z=H}$ (solid blue line) are the instantaneous $x y$-plane averaged wall shear stresses. $(b-g)$ Here $\omega_{y}^{y}$ overlaid by the velocity vector $\left(u^{y}, w^{y}\right) / U$. (h-m) Correspond to the same flow fields in $(b-g)$, respectively, but show the instantaneous streamwise velocity field near the top wall at $z / H=0.96$; the solid green line locates $u=0$, enclosing the flow reversal caused by the quasi-two-dimensional cyclone. The circled red arrow on the top right indicates the system rotation direction.

the formation of coherent large-scale circulations. These circulations are identified by the $y$-averaged spanwise vorticity $\omega_{y}^{y}=\left(\partial u^{y} / \partial z-\partial w^{y} / \partial x\right)$. Regions of high vorticity, hence strong circulation coincide with the regions of high Coriolis force. We can show this by taking the divergence of momentum equation $(2.1 b)-(2.1 d)$,

$$
\partial_{\tilde{x}_{i} \tilde{x}_{j}}\left(\tilde{u}_{i} \tilde{u}_{j}\right)=-\tilde{\nabla}^{2} \tilde{p}+R o^{-1} \tilde{\omega}_{y}+\partial_{\tilde{z}} \tilde{\theta} \quad i, j=1,2,3 .
$$

Equation (3.2) shows that the Coriolis term $R o^{-1} \tilde{\omega}_{y}$ is strong anywhere that $\widetilde{\omega_{y}}$ is large. In total, two circulations are formed: a strong cyclone near the top wall (marked with $\times$, green, corotating with the system) and a weak anticyclone in the bulk (marked with + , red, counter-rotating to the system). In figure $9(h-m)$, we observe that a cyclone is a quasi-two-dimensional roller that elongates in the spanwise direction. The $z$-location of the cyclone does not change with time, but it travels in the $x$-direction. The terms cyclone and anticyclone are widely used in rotating flows to identify coherent corotating or counter-rotating structures. However, they do not represent the shape of structures, e.g. columnar, plume-like or roll-like. The flow structures that we observe here as a large-scale concentrated cyclone accompanied by a field of weak anticyclones are also seen in rot_RB_slip with free-slip boundaries (Favier et al. 2014; Guervilly et al. 2014). Also, recent study of rot_RB_wall with no-slip boundaries (Aguirre Guzmán et al. 2020) 

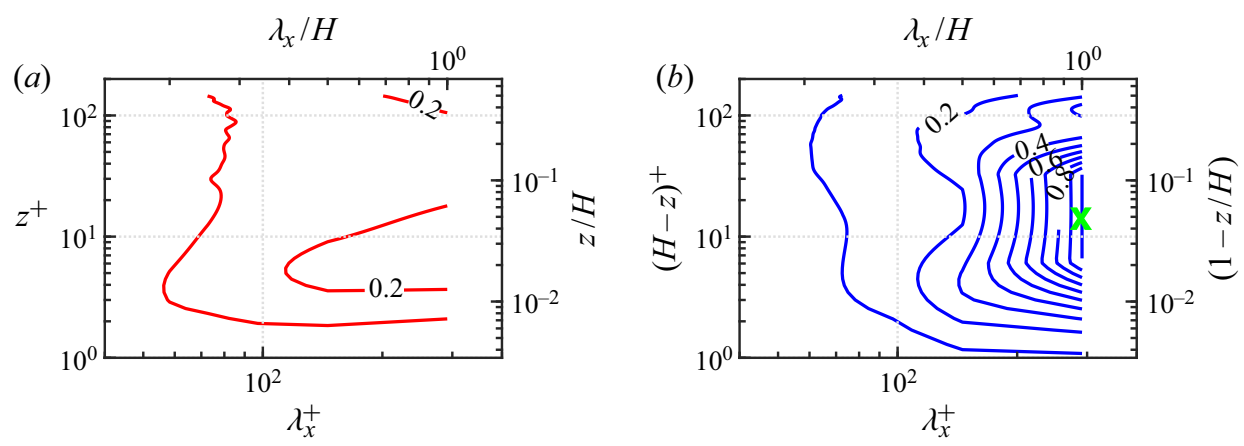

FIGURE 10. Streamwise premultiplied spectra for the $u$-velocity $k_{x} E_{u u}\left(k_{x}\right) / U^{2} \times 10^{2}$, where $\lambda_{x}=2 \pi / k_{x}$, at $R a=10^{8}$ and $R o^{-1}=1.0>R o_{\text {opt }}^{-1}=0.8$, the case visualised in figure 9 . Here (a) $0 \leq z / H \leq 0.5$ and $(b) 0 \leq(1-z / H) \leq 0.5$. The contour levels are increasing from 0.1 to 1.0 with an increment of 0.1 . The most energetic scale, marked with a green $\times$ in panel $(b)$, highlights the energetic cyclone near the top wall.

reveals such structures at $R a \gtrsim 10^{9}$ and $R o^{-1} \gtrsim 10$. However, these structures are not seen in rot_RB_cyl so far (Kunnen et al. 2011; Favier \& Knobloch 2020; Shishkina 2020; de Wit et al. 2020; Zhang et al. 2020).

To study the energy content of cyclones and anticyclones, in figure 10 we plot the premultiplied spectra of the case from figure 9 , at $R a=10^{8}$ and $R o^{-1}=1.0$. The most energetic scale (marked with $\times$, green in figure 10b) has the wavelength of $\lambda_{x}=H$ and is located at $(1-z / H) \simeq 0.05(z / H \simeq 0.95)$. This energetic scale is cyclone, marked with $(\times$, green) in figure $9(b-g)$. On the other hand, the energy content of anticyclone (located at $z / H \simeq 0.5$ ) is as low as the background turbulence. Such a clear symmetry breaking in favour of cyclone has been reported at moderately low Rossby numbers (in the order of unity), in rot_RB_slip (Favier et al. 2014; Guervilly et al. 2014), forced homogeneous rotating turbulence (Smith \& Waleffe 1999; Smith \& Lee 2005) and decaying homogeneous rotating turbulence (Bartello, Métais \& Lesieur 1994; Morize, Moisy \& Rabaud 2005; Moisy et al. 2011). Bartello et al. (1994), Morize et al. (2005) and Favier et al. (2014) studied the probability density function of vorticity. They concluded that the prevalence of cyclones is due to the dominance of cyclonic small scales.

\subsection{Local 'turbulence' Rossby number}

In our system, cyclones and anticyclones are formed at different transitional $R o^{-1}$, depending on $R a$. Also, at a fixed $R a$, the transitional $R o^{-1}$ depends on the rotating system. For example, in Favier et al. (2014) at $R a=10^{7}$ the transitional $R o^{-1}$ is 3.1 (while it is 1.2 in our system), and at $R a=10^{8}$ it is 1.8 (and 1.0 in our system). In both systems, the transitional $R o^{-1}$ decreases as $R a$ increases, but the transitional values are different. Favier et al. (2014) consider rotating Rayleigh-Bénard convection with free-slip hot and cold boundaries (rot_RB_slip in table 1), while we consider centrifugal convection (figure 1). The $R a$ and system dependency of transitional $R o^{-1}$ is because $R o^{-1} \equiv 2 \Omega H / U$ characterises the Coriolis force over the entire system. However, the Coriolis force is locally distributed differently, depending on $R a$ and the rotating system. Therefore, the formation of a cyclone as a local phenomenon, must be characterised based on a local measure of inverse Rossby number $\left(R o_{L}^{-1}\right)$. 
Study

Flow set-up

Transitional $R o_{L}^{-1}$

$\begin{array}{lcc}\text { Hopfinger, Browand \& Gagne (1982) } & \text { Cylindrical tank with oscillating grid } & \simeq 5.0 \\ \text { Godeferd \& Lollini (1999) } & \text { A horizontally infinite domain with forcing } & \simeq 3.0-5.0 \\ \text { Sugihara, Migita \& Honji (2005) } & \text { Rectangular tank with oscillating grid } & \simeq 5.5 \\ \text { Kinzel } \text { et al. } \text { (2011) } & \text { Rectangular tank with oscillating grid } & \simeq 5.0\end{array}$

TABLE 3. Summary of the previous studies that have reported the transitional local inverse Rossby number $R o_{L}^{-1}$, based on turbulent velocity scale and integral length scale. All the studies are experimental except Godeferd \& Lollini (1999).

(a)

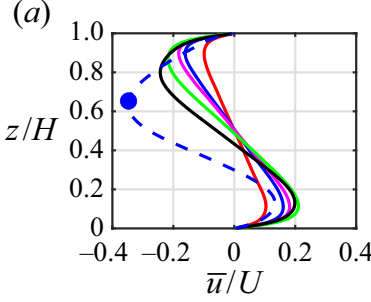

(e)

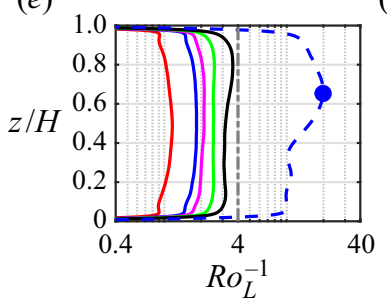

(b)

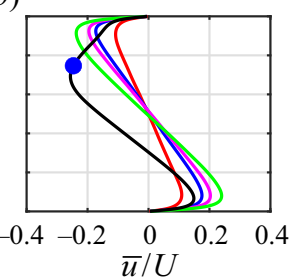

$(f)$

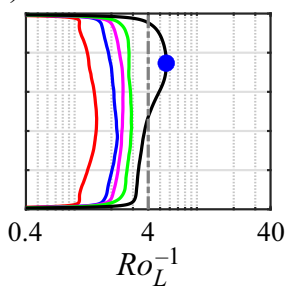

(c)

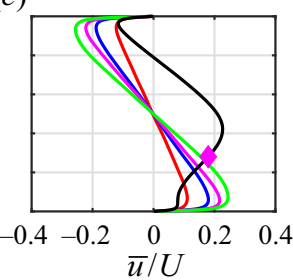

$(g)$

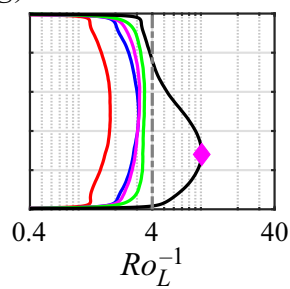

(d)

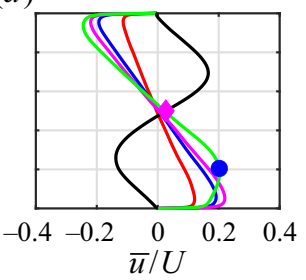

(h)

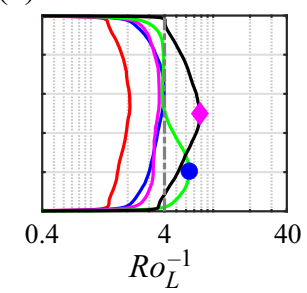

FIGURE 11. Profiles of the plane and time averaged streamwise velocity $\bar{u}(a-d)$ and local inverse Rossby number $R o_{L}^{-1} \equiv 2 \Omega \overline{\mathcal{K}} / \overline{\varepsilon_{u}}(e-h)$, at $R a=10^{7}(a, e), 10^{8}(b, f), 10^{9}(c, g)$ and $10^{10}$ $(d, h)$. Here $R o^{-1}=0.3$ (solid red line), 0.5 (solid blue line), 0.6 (solid magenta line), 0.8 (solid green line), 1.0 (solid black line) and 2.0 (dashed blue line). The vertical dashed-dotted grey line in the bottom row locates the transitional $R o_{L}^{-1}=4.0$, beyond which the cyclone is formed. All the $\bar{u}$ profiles that are deformed from their antisymmetric shape are marked with ( $\bullet$, blue; $\diamond$ hot magenta), as well as their $R o_{L}^{-1}$ profiles. Those marked with a blue $\bullet$ are partially deformed (still preserve their $\mathcal{S}$ shape). Those marked with a hot magenta $\checkmark$ are completely deformed. The markers are placed at the maximum $R o_{L}^{-1}$.

Hopfinger et al. (1982) defined $R o_{L}^{-1}$ based on local turbulent velocity scale and integral length scale. They observed the formation of cyclones when locally $R o_{L}^{-1}$ increases to approximately 5. Following Hopfinger et al. (1982), other studies (table 3) made the same observation at nearly the same transitional $R o_{L}^{-1}$. The studies in table 3 are both experimental and numerical, and consider different rotating systems. However, they all report a transitional $R o_{L}^{-1}$ close to 5 . In the rest of this subsection, first, we examine whether there is a unified transitional $R o_{L}^{-1}$ for our rotating system, independent of $R a$. Then, by taking the advantage of this unified value, we attempt to arrive at a relation between $R o_{o p t}^{-1}$ (before the cyclone formation) and $R a$. We define local 'turbulence' inverse 

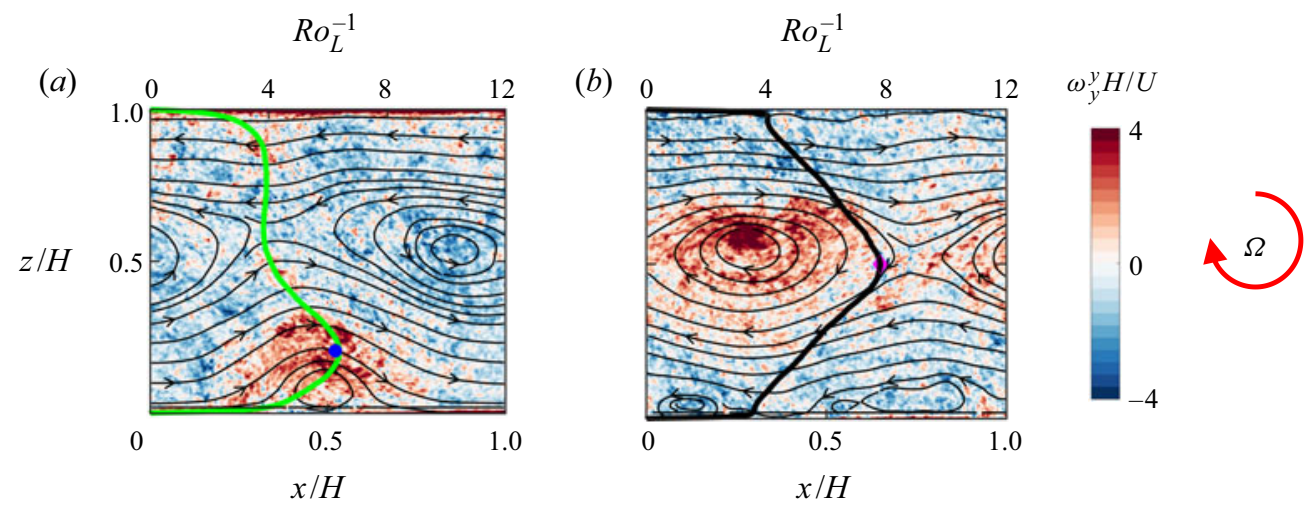

FIGURE 12. Instantaneous visualisation of $\omega_{y}^{y}=\left(\partial u^{y} / \partial z-\partial w^{y} / \partial x\right)$, spanwise-averaged spanwise vorticity, overlaid by the streamlines of $\left(u^{y}, w^{y}\right) / U$ and local inverse Rossby number $R o_{L}^{-1} \equiv 2 \Omega \overline{\mathcal{K}} / \overline{\varepsilon_{u}}$, at $R a=10^{10}$ and $(a) R o^{-1}=0.8$ and $(b) R o^{-1}=1.0$. Panels $(a, b)$ correspond to the profiles (solid green line) and (solid black line) in figure $11(d, h)$, respectively. The vertical and bottom horizontal axes are the wall-normal $z / H$ and streamwise $x / H$ coordinates, respectively, for the vorticity field and velocity vector. The top horizontal axis is $R o_{L}^{-1}$ for the overlaid profiles of $\mathrm{Ro}_{L}^{-1}$ versus $z / H$. The circled red arrow on the right-hand side indicates the system rotation direction.

Rossby number $R o_{L}^{-1} \equiv(2 \Omega \overline{\mathcal{K}}) / \overline{\varepsilon_{u}}$ based on eddy turnover time $\overline{\mathcal{K}} / \overline{\varepsilon_{u}}$, where $\overline{\mathcal{K}}$ is the turbulent kinetic energy.

In figure 11 we plot the profiles of $R o_{L}^{-1}$ at $R a=10^{7}$ (figure 11e) to $10^{10}$ (figure $11 \mathrm{~h}$ ), and $R O^{-1}=0.3$ (solid red line) to 2.0 (dashed blue line). We also add the plane and time averaged streamwise velocity profiles $\bar{u}$ (figure $11 a-d$ ). We aim to see if there is any relation between $R o_{L}^{-1}$ and wind breaking, i.e. when the antisymmetric $\bar{u}$ profile is deformed. At each $R a$, we mark the deformed $\bar{u}$ profiles and their corresponding $R o_{L}^{-1}$ profiles. The profiles marked with a blue $\bullet$ indicate the stage where the $\bar{u}$ profile still preserves its $(\mathcal{S})$ shape, while the profiles marked with a hot magenta indicate the stage where the $\bar{u}$ profile is completely deformed. We compare these two stages at $R a=10^{10}$ in figure 12. These stages correspond to the solid green line and solid black line in figure $11(d, h)$. The stage marked with a blue • (figure $12 a$ ) is slightly beyond $R o_{o p t}^{-1}$ and cyclone is near the wall. The stage marked with a hot magenta $\downarrow$ (figure $12 b$ ) is further beyond $R o_{o p t}^{-1}$ and the cyclone has migrated to the bulk. As a result, the $\bar{u}$ profile direction is reversed (solid black line in figure $11 d$ ). We see similar trend in the $\bar{u}$ profile at $R a=10^{8}$ (figure $8 a$ ). The reversal in $\bar{u}$ from $R o^{-1}=1$ (solid black line) to 20 (solid grey line) is due to the migration of cyclone to the bulk.

Considering all the marked $R o_{L}^{-1}$ profiles in figure 11(e-h), wind breaking starts (cyclone appears) once $R o_{L}^{-1} \gtrsim 4.0$. This transitional $R o_{L}^{-1} \simeq 4.0$ is close to the value of 5.0 reported in the literature (table 3 ). The region of $R o_{L}^{-1}>4$ locates a cyclone (figure 12) with maximum $R o_{L}^{-1}$ near the core of the cyclone. Slightly beyond $R o_{o p t}^{-1}$ (marked with -, blue in figure 11), the maximum $R o_{L}^{-1}$ also coincides with the peak of $\bar{u}$. Because the cyclone is small (figure 12a), hence its core (the maximum $R o_{L}^{-1}$ ) and its edge (the peak of $\bar{u}$ ) are close to each other. Further beyond $R o_{o p t}^{-1}$ (marked with $\downarrow$, hot magenta in figure 11), the maximum $R o_{L}^{-1}$ does not coincide with the peak of $\bar{u}$. Because the cyclone is large (figure $12 b$ ), hence its core and its edge are distant from each other. 

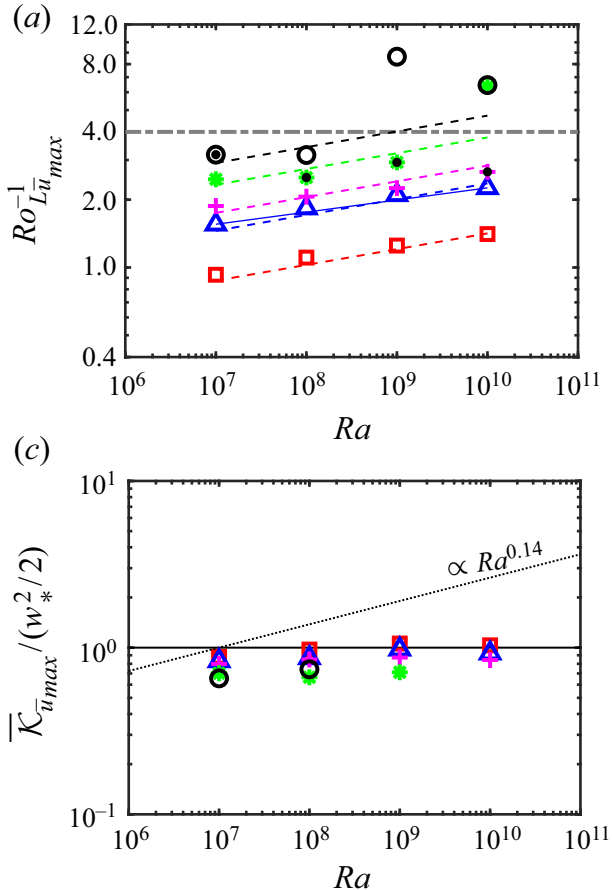

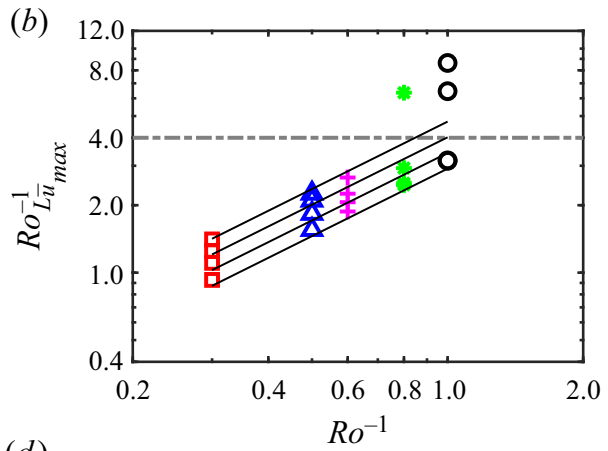

$(d)$

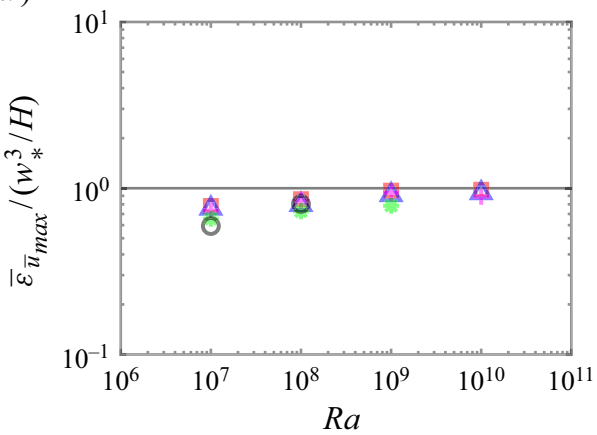

FIgURE 13. Variation of $R o_{L}^{-1}$ at the maximum $\bar{u}\left(R o_{L_{\bar{u}_{m a x}}}^{-1}\right)$ : $(a)$ as a function of $R a$ for different $R o^{-1}$ values and $(b)$ as a function of $R o^{-1}$ for different $R a$ values. The horizontal line (dashed-dotted grey line) in panels $(a, b)$ mark $R o_{L_{\bar{u}_{\max }}}^{-1}=4$, above which the cyclone forms. The symbols are from the simulations at $R o^{-1}=0.3$ ( $\square$, red), 0.5 ( $\triangle$, blue), 0.6 (+, magenta),

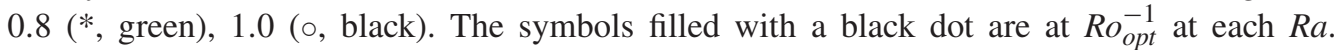
The fitting dashed lines in panel $(a)$ and solid lines in panel $(b)$ are the theoretical relation $R o_{L_{\bar{u}} \max }^{-1} \simeq 0.94 R a^{0.07} R o^{-1}$. This relation is derived based on the scaling arguments for $\overline{\mathcal{K}}_{\bar{u}_{\text {max }}} \propto$ $w_{*}^{2}, \bar{\varepsilon}_{\bar{u}_{\max }} \propto w_{*}^{3} / H, N u \propto R a^{0.29}$ and $\operatorname{Pr}=0.7$ (see the text), where $w_{*} \equiv \kappa / H(\operatorname{PrNuRa})^{1 / 3}$. Panels $(c, d)$ verify the scaling arguments for $\overline{\mathcal{K}}_{\bar{u}_{\text {max }}}$ and $\bar{\varepsilon}_{\bar{u}_{\text {max }}}$, respectively. For clarity, in $(c, d)$ we only show the data points with $R o_{L}^{-1}<4$ (below dashed-dotted grey line in panel $a$ ). The dotted line in panel $(c)$ indicates the scaling $\overline{\mathcal{K}}_{\bar{u}_{\max }} \propto w_{*}^{2} R a^{0.14}$ based on the correction by Xie et al. (2019).

Figure 11(e-h) shows that $R o_{L}^{-1}$ monotonically increases, by increasing $R a$ or $R o^{-1}$, up to $R o_{L}^{-1} \simeq 4$.0. We reaffirm this behaviour in figure 13 , plotting $R o_{L}^{-1}$ at the maximum $\bar{u}\left(R o_{\bar{L}_{\max }}^{-1}\right)$ versus $R a$ (figure $13 a$ ) and $R o^{-1}$ (figure 13b). Here $R o_{\bar{u}_{\max }}^{-1}$ increases with a power of $R a$ or $R o^{-1}$ up to $R o_{L_{\bar{m} \max }}^{-1} \simeq 4.0$ (marked with a dashed-dotted grey line in figure $13 a, b)$. We can support this power-law behaviour through scaling arguments for

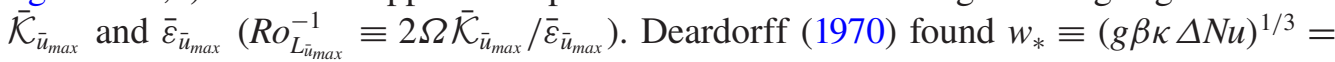
$\kappa / H(\operatorname{PrNuRa})^{1 / 3}$ as a suitable velocity scale for the r.m.s. of wall-normal velocity fluctuations $w_{r m s}$ in Rayleigh-Bénard convection. Xie, Hu \& Xia (2019) observed that $w_{r m s} / w_{*}$ weakly depends on $R a\left(\sim R a^{0.07 \pm 0.02}\right)$. Here, our data of $\overline{\mathcal{K}}_{\bar{u}_{\max }}$ scale well with $w_{*}^{2}$, without $R a$ correction (figure $13 c$ ). One possibility could be due to our unbounded (periodic) domain compared with the bounded (cylindrical or cubic) domains in Xie et al. (2019). Another possibility could be due to the Coriolis force that is absent in 
Rayleigh-Bénard convection of Xie et al. (2019). Our data of $\bar{\varepsilon}_{\bar{u}_{\max }}$ scale well with $w_{*}^{3} / H=v^{3} H^{-4} \mathrm{NuRaPr}^{-2}$ (figure 13d). Because the volume and time averaged dissipation rate is $\left\langle\varepsilon_{u}\right\rangle \simeq w_{*}^{3} / H$ in the inertia-dominated regime $(N u \gg 1)$, see $\S 2.2$. With these scales for $\overline{\mathcal{K}}_{\bar{u}_{\max }}$ and $\bar{\varepsilon}_{\bar{u}_{\max }}$, we obtain $R o_{L_{\bar{u}_{\max }}^{-1}} \propto \mathrm{Pr}^{1 / 6} R a^{1 / 6} R o^{-1} N u^{-1 / 3}$. Considering $\mathrm{Nu}$ versus $R a$ (figure 5) for $R o^{-1}<1$, it nearly falls into the classical regime scaling, i.e. $N u \propto R a^{0.29}$ (Scheel \& Schumacher 2016). Substituting for $N u$ and $P r=0.7$, we obtain $R o_{L_{\bar{u} \max }^{-1}}^{-1} \propto 0.94 R a^{0.07} R o^{-1}$. Figures 13(a), dashed lines, and 13(b), solid lines, show the good agreement between this power-law relation and the simulation data up to $R o_{L}^{-1} \leq 4$.

We can predict $R o_{o p t}^{-1}$ at each $R a$ using this power-law. The data points at $R o_{o p t}^{-1}$ (filled with black dots in figure $13 a$ ), fall at $R o_{L_{\bar{u}_{\max }}}^{-1} \simeq 3.0$. After substituting for $R o_{L_{\bar{u}_{\max }}}^{-1}=3.0$ and $R o^{-1}=R o_{o p t}^{-1}$ in the power-law relation and some recasting, we arrive at $R o_{o p t}^{-1} \simeq$ $3.19 R a^{-0.07}$. This relation confirms the slight variation of $R o_{o p t}^{-1}$ with $R a$ (figure $6 a$ ).

Our approach in this subsection is similar to the one by King et al. (2009); to identify the transition from the buoyancy (inertia) dominated to the Coriolis dominated regime. King et al. (2009) focus on rot_RB_wall (table 1), while we consider centrifugal convection. Both in King et al. (2009) and here, we relate the transition to a local phenomenon. In King et al. (2009) the transition occurs in the Ekman layer. They explain the transition through the competition between the Ekman layer and thermal boundary layer. Here, the transition occurs in the bulk, i.e. the locale of cyclone. Therefore, we explain the transition through $R o_{L}^{-1} \equiv 2 \Omega \overline{\mathcal{K}} / \bar{\varepsilon}_{u}$, i.e. the competition between the system rotation time scale and the turbulence time scale.

\subsection{Flow behaviour at very large Coriolis force}

At the very large $R o^{-1}=20$ (figure $7 d, h$ ), turbulence is suppressed and the flow becomes two-dimensional and laminar-like. In figure 14 , we visualise this case over a period of $150 H / U$. In the top row, we visualise $\tilde{\theta}$ overlaid by the $(\tilde{u}, \tilde{w})$ streamlines. In the bottom row, we visualise the vorticity field $\tilde{\omega}_{y}=\left(\partial_{\tilde{z}} \tilde{u}-\partial_{\tilde{x}} \tilde{\mathcal{w}}\right)$. We locate the core of cyclones $(\checkmark$, green) and anticyclones $(\bullet$, red) based on the core of streamlines, i.e. $(\tilde{u}, \tilde{w}) \simeq(0,0)$. Figure 14 shows that at the very large $R o^{-1}$, the cyclone in the bulk and anticyclones near the walls become equally strong. The stabilisation of both cyclones and anticyclones at very large $R o^{-1}$ is observed in rotating Rayleigh-Bénard convection with free-slip boundaries (rot_RB_slip). For instance, refer to figure 3 in Stellmach et al. (2014) (Ra $\simeq$ $\left.2 \times 10^{11}, R o^{-1} \simeq 23\right)$ or figure 2 in Guervilly et al. (2014) $\left(R a \simeq 8 \times 10^{8}, R o^{-1} \simeq 7\right)$. However, in those studies turbulence is not completely suppressed. This difference might be due to their different flow set-up (rot_RB_slip in table 1) compared with our set-up (centrifugal convection).

In figure $14(b-g)$, the cyclone has almost a uniform zero temperature. Also, the anticyclones near the bottom and top walls have a uniform hot and cold temperature, respectively. To explain this phenomenon, in figure 15 we study different terms of $u$ - and $w$-momentum equations. The diffusion terms are negligible and the momentum balance is primarily between the pressure gradient (figure $15 c, g$ ) and Coriolis force (figure $15 d, h$ ). This balance is known as the geostrophic balance (Greenspan 1968), leading to the geostrophic regime. In this regime, there is a drop in $N u$ (King et al. 2009; Schmitz \& Tilgner 2009; Ecke \& Niemela 2014), also seen in our case at $R a=10^{8}$ from $R o^{-1}=1$ to 20 (o, black to $\nabla$, olive green, in figure 5 ). This regime also appears in rapidly rotating Rayleigh-Bénard convection, in all the common set-ups of this flow (table 1). To name a few for rot_RB_wall see Julien et al. (1996b), Kunnen et al. (2006) and King et al. $(2009,2012)$; for rot_RB_slip see Stellmach et al. (2014) and Guervilly et al. (2014); and 

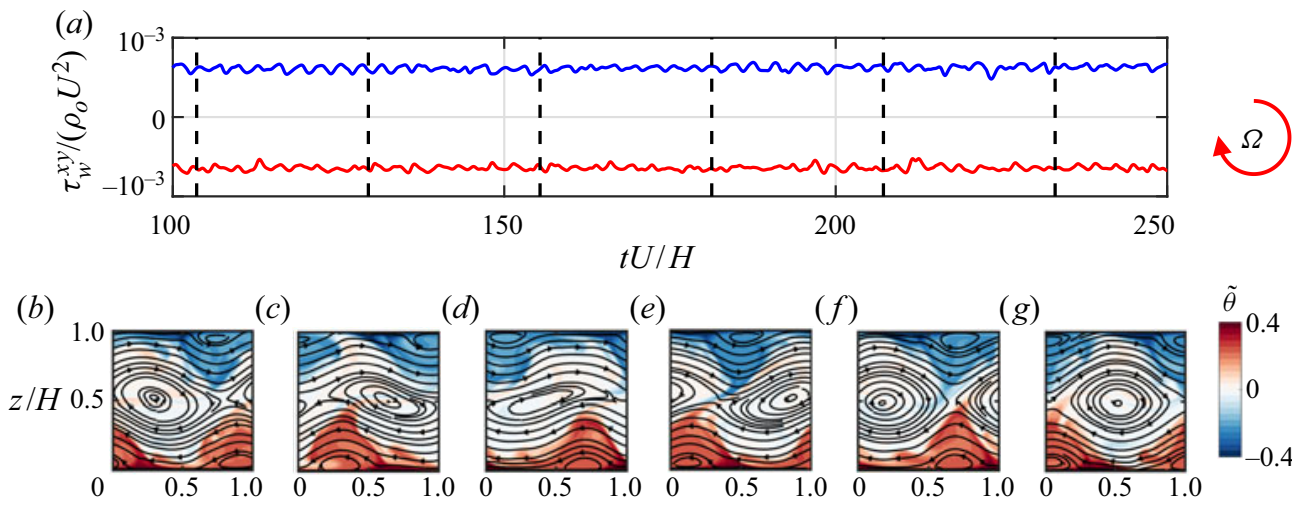

(c)

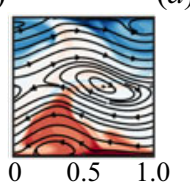

(d)

(e)
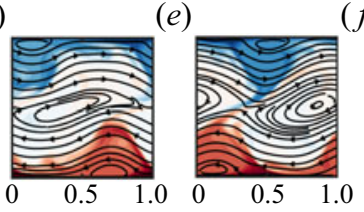

(f)

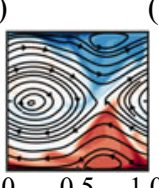

$(g)$
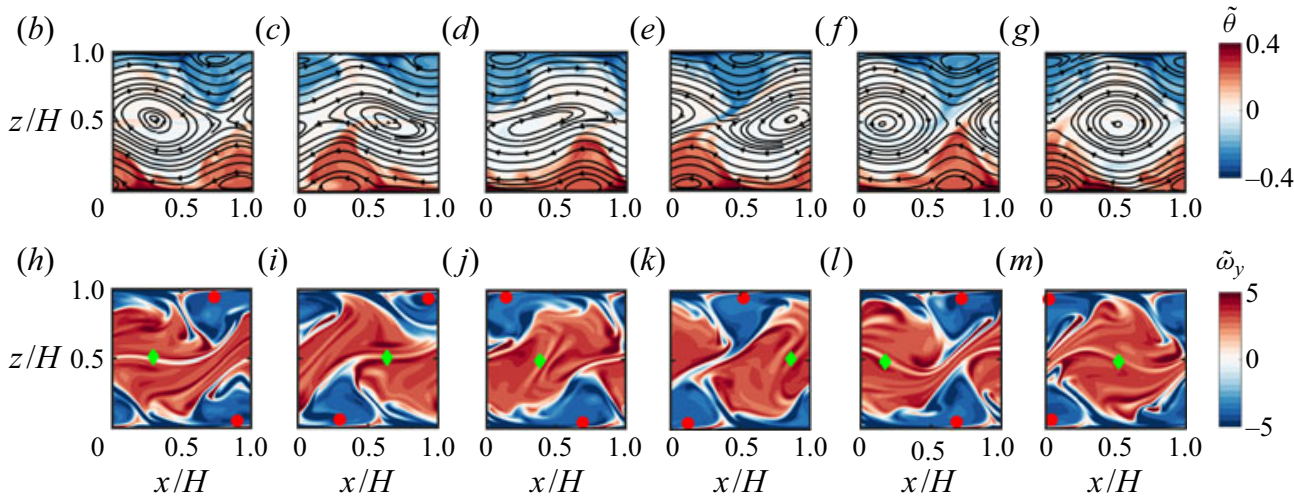

(j)

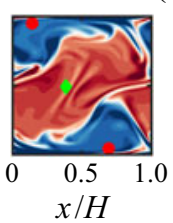

$(k)$

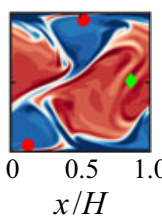

(l)

$(m)$
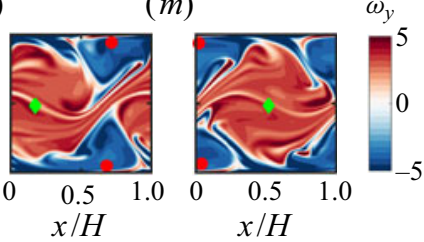

Figure 14. Flow visualisation at different times $t U / H$ for $R a=10^{8}, R o^{-1}=20$. Note that the flow is two-dimensional, i.e. depends on $x, z$ only. The visualisation times from panel $(b)$ to panel $(g)$ are indicated by vertical dashed lines in the plane-averaged wall shear stress $\tau_{w}^{x y}$ history. The solid red line and solid blue line are, respectively, the bottom and top wall, $\tau_{w}^{x y}$. (b-g) Temperature field $\tilde{\theta}=\theta / \Delta$ overlaid by the streamlines. $(h-m)$ Correspond to the same flow fields in $(b-g)$, respectively, but show the vorticity $\tilde{\omega}_{y}=(H / U)(\partial u / \partial z-\partial w / \partial x)$. The core of cyclone $\left(\diamond\right.$, green, $\left.\omega_{y}>0\right)$ and anticyclone $\left(\bullet\right.$, red, $\left.\omega_{y}<0\right)$ are marked based on $(u, w) \simeq(0,0)$, i.e. the core of streamlines. The circled red arrow on the top right indicates the system rotation direction.

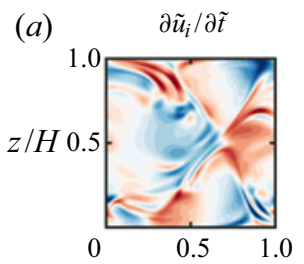

(b) $\quad \partial \tilde{u}_{i} \tilde{u}_{j} / \partial \tilde{x}_{j}$

(e)

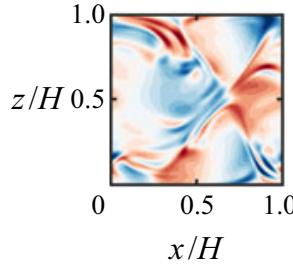

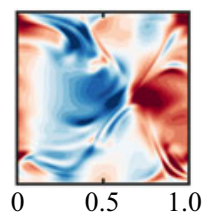

(c)

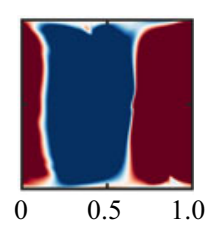

$(g)$

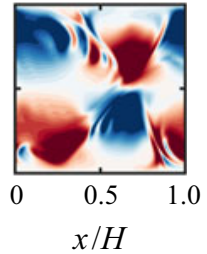

(d) $-R o^{-1} \epsilon_{i j k} \tilde{u}_{k}$

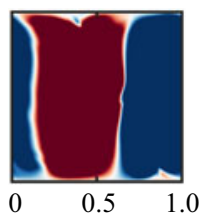

(h)

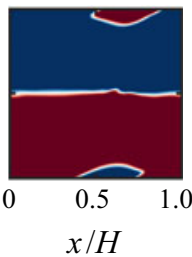

(i)

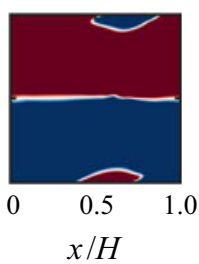

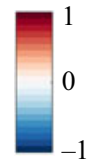

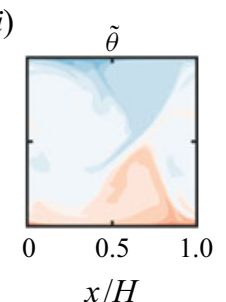

FIGURE 15. Terms of the $u$-momentum $(a-d)$ and $w$-momentum equation $(e-i)$ at $R a=10^{8}$ and $R o^{-1}=20$. Note that the flow is two-dimensional, i.e. depends on $x, z$ only. Here $(a, e) \partial \tilde{u}_{i} / \partial \tilde{t}$; $(b, f) \partial \tilde{u}_{i} \tilde{u}_{j} / \partial \tilde{x}_{j} ;(c, g)-\partial \tilde{p} / \partial \tilde{x}_{i} ;(d, h)-R o^{-1} \epsilon_{i j k} \tilde{u}_{k}$, where $\epsilon_{i j k}$ is the permutation tensor; and (i) $\tilde{\theta}$. The diffusion terms are negligible, i.e. $(\operatorname{Pr} / \operatorname{Ra})^{1 / 2}\left(\partial^{2} \tilde{u}_{i} / \partial \tilde{x}_{j}^{2}\right) \simeq O\left(10^{-2}\right)$. 
for rot_RB_cyl see Vorobieff \& Ecke (2002), Ecke \& Niemela (2014), Ecke (2015) and Rajaei, Kunnen \& Clercx (2017). However, the flow phenomenology evolves differently among these three systems (Kunnen et al. 2016; Zhang et al. 2020). In planetary flows, such as atmospheric or ocean circulations, $R o^{-1}$ can go beyond 10 (Kundu \& Cohen 1990) and geostrophic balance occurs.

Although the geostrophic balance is between the Coriolis force and pressure gradient, in our system we cannot ignore the transient (figure 15a,e) and advection terms (figure $15 b, f$ ), as figure 14 shows that this is an unsteady problem. Also we cannot ignore the buoyancy term (figure 15i), as there would be no flow without this term. We also assessed the terms of the temperature transport equation (2.1e). Similar to momentum, the diffusion terms are orders of magnitude smaller than the transient and advection terms. Therefore, the governing equations $((2.1 a)-(2.1 e))$ are simplified to

$$
\partial_{\tilde{x}} \tilde{u}+\partial_{\tilde{z}} \tilde{w}=0, \quad D_{\tilde{t}} \tilde{u}=-\partial_{\tilde{x}} \tilde{p}-R o^{-1} \tilde{w}, \quad D_{\tilde{t}} \tilde{w}=-\partial_{\tilde{z}} \tilde{p}+R o^{-1} \tilde{u}+\tilde{\theta}, \quad D_{\tilde{t}} \tilde{\theta}=0,
$$

where $D_{\tilde{t}}\langle\cdot\rangle=\partial_{\tilde{t}}\langle\cdot\rangle+\tilde{u} \partial_{\tilde{x}}\langle\cdot\rangle+\tilde{w} \partial_{\tilde{z}}\langle\cdot\rangle$ is the material derivative. The material derivative of a property implies the rate of change of that property as we track a cyclone or anticyclone. We recast $(3.3 a-d)$ in terms of stream function $\tilde{\psi}\left(\tilde{u}=\partial_{\tilde{z}} \tilde{\psi}, \tilde{w}=-\partial_{\tilde{x}} \tilde{\psi}\right)$ and $\tilde{\omega}_{y}$ as follows:

$$
\partial_{\tilde{x} \tilde{x}}^{2} \tilde{\psi}+\partial_{\tilde{z} \tilde{z}}^{2} \tilde{\psi}=\tilde{\omega}_{y}, \quad D_{\tilde{t}} \tilde{\omega}_{y}=-\partial_{\tilde{x}} \tilde{\theta}, \quad D_{\tilde{t}} \tilde{\theta}=0 .
$$

A similar set of equations is solved for quasi-geostrophic flows to study the interaction of cyclones and anticyclones with themselves (Reinaud, Dritschel \& Koudella 2003; Dritschel, Reinaud \& McKiver 2004; Reinaud \& Carton 2016) or with the wall (Deremble, Johnson \& Dewar 2017; Venaille 2020). Equation (3.4c) justifies figure $14(b-g)$; the temperature of cyclone and anticyclones does not change with time. Also, (3.4b) justifies the uniform $\tilde{\theta}$ within each cyclone and anticyclone. Considering figure $14(h-m)$, the vorticity of cyclone and anticyclones is materially conserved, i.e. $\left(D_{\tilde{t}} \tilde{\omega}_{y}\right)_{\text {cyclone }} \simeq$ $\left(D_{\tilde{t}} \tilde{\omega}_{y}\right)_{\text {anticyclone }} \simeq 0$. Therefore, from $(3.4 b)$ we expect $\left(\partial_{\tilde{x}} \tilde{\theta}\right)_{\text {cyclone }} \simeq\left(\partial_{\tilde{x}} \tilde{\theta}\right)_{\text {anticyclone }} \simeq 0$, i.e. $\tilde{\theta}$ is uniform within each cyclone or anticyclone.

\subsection{Towards logarithmic boundary layers}

In $\$ 3.1$ we showed the remarkable feature of this centrifugal system in forming a bidirectional wind with the maximum momentum at $R o_{o p t}^{-1}$, presumably helping the boundary layer transition to the turbulent Prandtl-von Kármán type, as hypothesised by Kraichnan (1962). In this subsection, we demonstrate this feature by studying the structure of the wind as $R a$ increases. The value of $R o_{o p t}^{-1}$ changes with $R a$, from 1.0 at $R a=10^{7}$, to 0.6 at $R a=10^{10}$ (figure $6 b$ ). Here, we fix $R o^{-1}$ at 0.8 , a value slightly smaller or larger than $R o_{o p t}^{-1}$, so that the system still generates a high momentum wind. In figure 16 , we study the mean velocity and temperature profiles and r.m.s. of their fluctuations from the top wall to the domain midheight, as $R a$ increases. To study the distance to a turbulent boundary layer, in figure 16 in addition to the law of the wall for Prandtl-von Kármán type boundary layer (dashed-dotted blue line), we overlay the mean and r.m.s. velocity profiles by their counterparts from the DNS of turbulent channel flow (solid red line) at $R e_{\tau} \simeq 180$ (Lee \& Moser 2015), based on channel half-height, and we overlay the temperature profiles by their counterparts from the DNS of turbulent channel flow with passive scalar (dotted red line) at $R e_{\tau}=180$ and $\operatorname{Pr}=0.71$ (Kim \& Moin 1989). We choose this value of $R e_{\tau}$ for comparison, because in our system at the highest $R a=10^{10}$ (solid black line in figure 16a), 

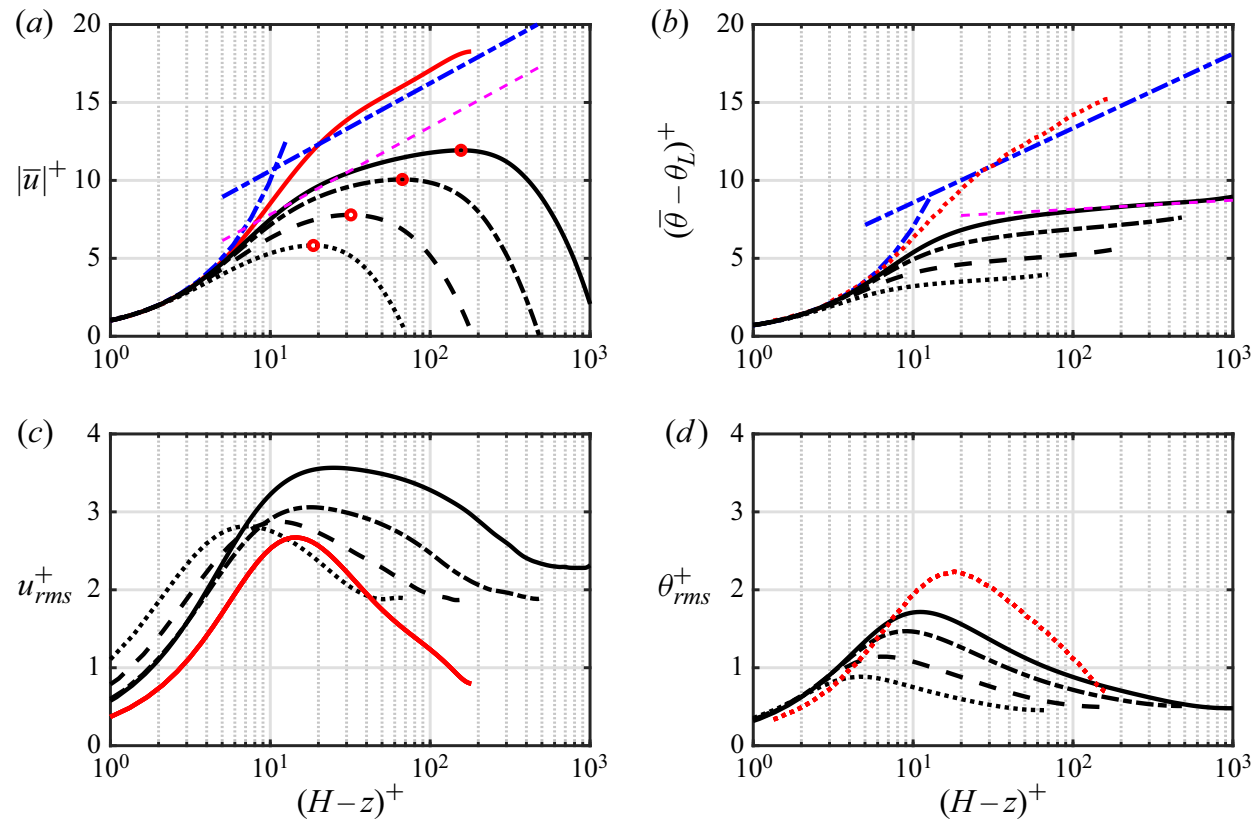

FigURE 16. Profiles of the $(a)$ absolute mean velocity $|\bar{u}|,(b)$ mean temperature $\left(\bar{\theta}-\theta_{L}\right)=$ $(\bar{\theta}+\Delta / 2),(c)$ r.m.s. of velocity fluctuation $u_{r m s}=\sqrt{\overline{\overline{u^{\prime 2}}}}$ and $(d)$ r.m.s. of temperature fluctuation $\theta_{r m s}=\sqrt{\overline{\theta^{\prime 2}}}$ from the top wall to the domain midheight, $0 \leq(H-z) \leq H / 2$, at $R o^{-1}=0.8$ and $R a=10^{7}$ (dotted black line), $10^{8}$ (dashed black line), $10^{9}$ (dashed-dotted black line) and $10^{10}$ (solid black line). We normalise $|\bar{u}|$ and $u_{r m s}$ by the top wall friction velocity $u_{\tau_{\text {top }}}$. We normalise $\left(\bar{\theta}-\theta_{L}\right)$ and $\theta_{\text {rms }}$ by $\theta_{\tau_{\text {top }}}=-\kappa\left(\mathrm{d} \bar{\theta} /\left.\mathrm{d} z\right|_{z=H}\right) / u_{\tau_{\text {top }}}$. In panel $(a)$, we plot law of the wall (dashed-dotted blue line) in the viscous sublayer $\bar{u}^{+}=z^{+}$and log layer (Prandtl-von Kármán profile) $\bar{u}^{+}=(1 / 0.41) \ln \left(z^{+}\right)+5.2$ (Yaglom 1979). In panel $(b)$, we plot Prandtl-von Kármán type temperature profile for fully turbulent flow (dashed-dotted blue line) in the viscous sublayer $\bar{\theta}^{+}=\operatorname{Prz}^{+}$, and log layer $\bar{\theta}^{+}=(0.85 / 0.41) \ln \left(z^{+}\right)+3.8$ (Yaglom 1979). In panels $(a, c)$, we add DNS of channel flow by Lee \& Moser (2015) (solid red line) at $R e_{\tau} \simeq 180$. In panels $(b, d)$, we add DNS of channel flow by Kim \& Moin (1989) (dotted red line) at $R e_{\tau} \simeq 180$ with passive scalar at $\operatorname{Pr}=0.71$. The logarithmic fitting line (dashed hot magenta line) has the slope $1 / 0.41$ in panel $(a)$ and has the slope $1 / 4.0$ in panel $(b)$. In panel $(a)$, the velocity boundary layer thickness is located by $(\circ$, red $)$.

$R e_{\tau}$ reaches approximately 155 based on the velocity boundary-layer thickness. We define the boundary-layer thickness where $|\bar{u}|^{+}$is maximum.

The $\bar{u}^{+}$profiles (figure 16a) progress towards Prandtl-von Kármán (logarithmic) behaviour as $R a$ increases. Nevertheless, full collapse on the logarithmic law, corresponding to a fully turbulent wall-bounded flow, is not reached yet at $R a=10^{10}$ (solid black line in figure 16a). A narrow logarithmic region (dashed magenta line) with a slope of $1 / 0.41$ starts to appear from $R a=10^{9}$ (dashed-dotted black line in figure 16a). This is consistent with the two-dimensional Rayleigh-Bénard simulation of Zhu et al. (2018) who observed the emergence of the logarithmic slope $1 / 0.41$ in the mean velocity profiles before the ultimate regime $\left(R a<10^{13}\right)$. They also observed a much shallower logarithmic slope (approximately 1/4) in their temperature profiles compared with what is expected in a fully turbulent boundary layer $(0.84 / 0.41 \simeq 1 / 0.48)$. We observe similar behaviour in 


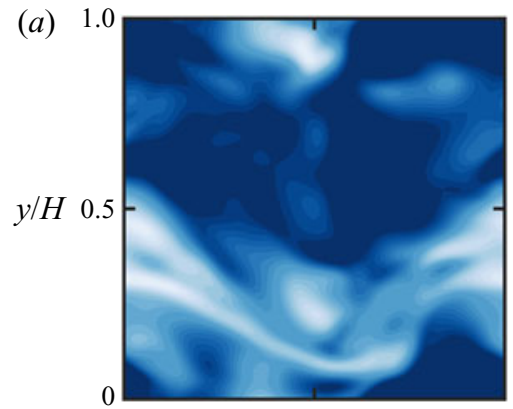

(b)
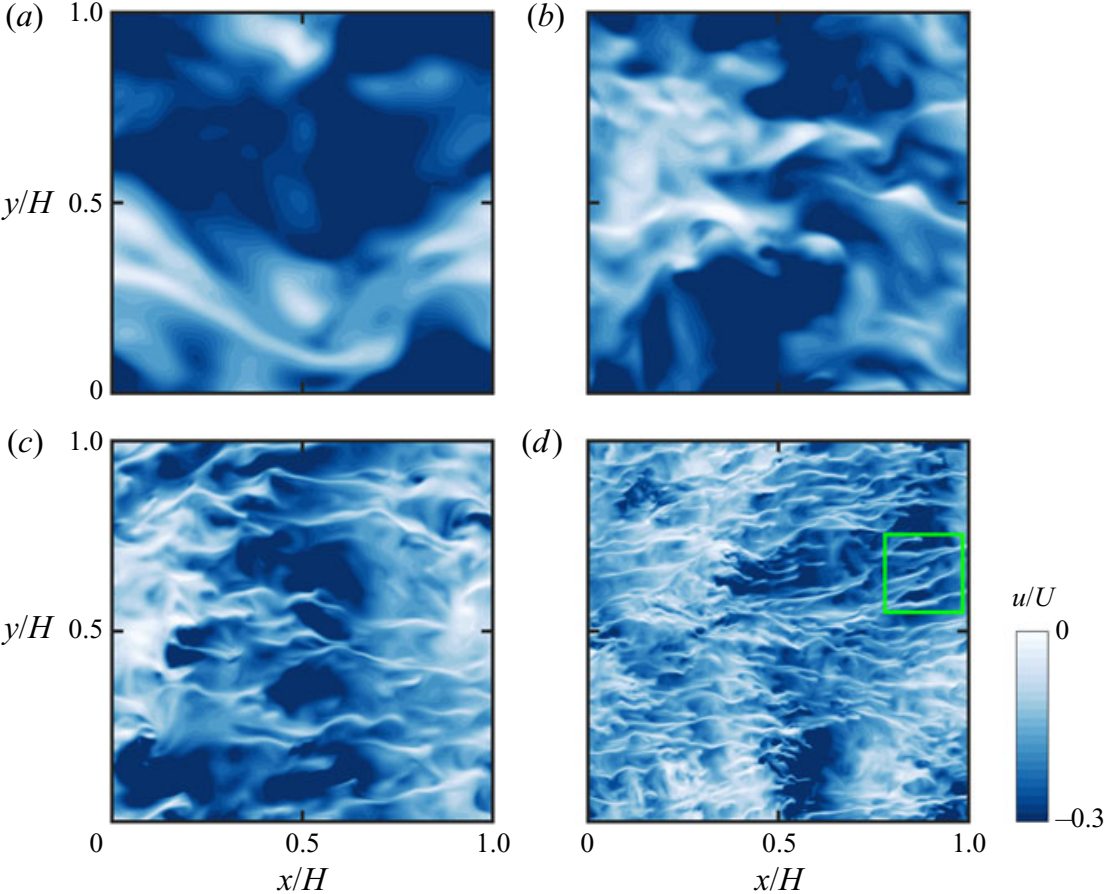

FIGURE 17. Instantaneous fields of $u$ at a distance of 15 wall units from the top wall at $R o^{-1}=$ 0.8 and $R a=10^{7}(a), 10^{8}(b), 10^{9}(c)$ and $10^{10}(d)$. The green square in panel $(d)$ encloses an area of $500 \times 500$ wall units, encompassing approximately five streaks in the spanwise direction.

the $\bar{\theta}^{+}$profiles in figure $16(b)$, considering the fitting lines (dashed magenta line) with a slope of $1 / 4.0$.

From figure $16(a, b)$, it appears that the mean velocity profiles approach the fully turbulent counterpart faster than the mean temperature profiles. Similar behaviour is seen in the r.m.s. profiles (figure $16 c, d$ ). Both $u_{r m s}$ and $\theta_{r m s}$ profiles yield an inner peak near the wall. The inner peak in the $u_{r m s}^{+}$profiles (figure 16c) is approximately 3.0 at all values of $R a$, which is close to the DNS of channel flow (solid red line). As $R a$ increases, the location of the $u_{r m s}^{+}$inner peak approaches the one corresponding to the fully turbulent channel flow (solid red line). On the other hand, the inner peak of the $\theta_{r m s}^{+}$profiles (figure $16 d$ ) is smaller than the fully turbulent counterpart (dotted red line), even at the highest value of $R a=10^{10}$ (solid black line in figure $16 d$ ).

Our observations from figure 16 give indications for the onset of the transition to turbulence in the velocity boundary layer at $R a=10^{10}$. We visualise this transition in figure 17, showing the instantaneous field of $u$ at 15 wall units away from the top wall. We observe the streak-like structures at $R a=10^{10}$ (figure $17 d$ ), similar to a fully turbulent boundary layer. The green square with an area of $500 \times 500$ wall units highlights the approximately 100 wall units spanwise spacing between the near-wall streaks, as in a turbulent wall-bounded flow (Kline et al. 1967). We also see the transition to turbulence in the spectral distribution of Reynolds shear-stress near the top wall (figure 18). We plot the spectrograms of $\overline{u^{\prime} w^{\prime}}$ at $R o^{-1}=0.8$ and $R a$ from $10^{7}$ (figure $18 a$ ) to $10^{10}$ (figure $18 d$ ). In figure $18(d)$ we overlay the spectrogram by the one from the DNS of turbulent channel flow (Lee \& Moser 2015) at $R e_{\tau} \simeq 180$ (solid red line). As we observe, the spectral distribution of the near-wall region at $R a=10^{10}$ is similar to that of a turbulent channel flow. The spacing of 100 wall units between the energetic near-wall streaks (figure $17 d$ ) 
(a)

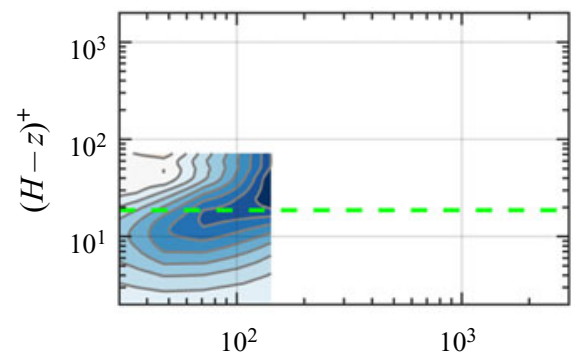

(c)

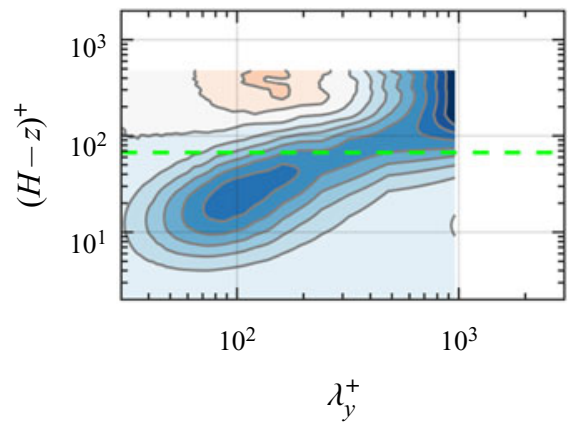

(b)

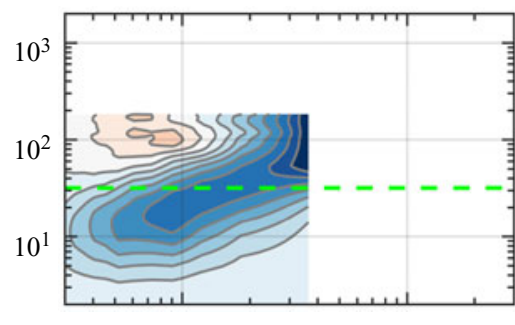

$10^{3}$

(d)

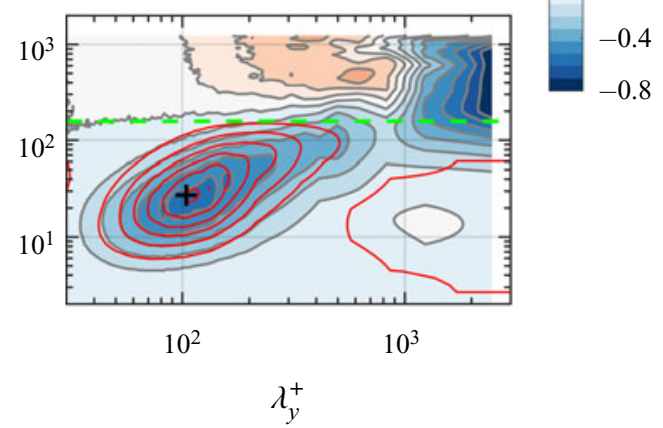

FIGURE 18. Premultiplied spectra of Reynolds shear stress $k_{y} E_{u w}\left(k_{y}\right) / u_{\tau}^{2}$ for $0 \leq(1-z / H) \leq$ 0.5 , where $\lambda_{y}=2 \pi / k_{y}$, for the cases shown in figure 17 at $R o^{-1}=0.8$ and $R a=(a) 10^{7},(b) 10^{8}$, (c) $10^{9}$ and $(d) 10^{10}$. The horizontal line in each plot marks the height where $|\bar{u}|$ is maximum in figure $16(a)$. Panel $(d)$ is overlaid by $k_{y} E_{u w}\left(k_{y}\right) / u_{\tau}^{2}$ from the DNS of channel flow (Lee \& Moser 2015) at $R e_{\tau} \simeq 180$ (solid red line). The near-wall energetic mode is marked by a + , corresponding to the near-wall streak-like structures in figure $17(d)$. The contour levels increase from -0.8 to 0.8 with an increment of 0.1 .

can also be interpreted from the near-wall energetic mode (marked by ' + ' in figure $18 d$ ), at $(H-z)^{+} \simeq 20$ and $\lambda_{y}^{+} \simeq 100$. This subsection supports our earlier conjecture that the bidirectional wind helps the boundary layer transition to turbulent, hence the transition to the ultimate regime.

\section{Conclusions}

Detailed DNS and analysis of centrifugal buoyancy-driven convection has been carried out with a focus on the effect of the Coriolis force. The inverse Rossby number $R o^{-1}$ was varied from zero to 20 , the Rayleigh number $R a$ from $10^{7}$ to $10^{10}$ and the Prandtl number was fixed at $\operatorname{Pr}=0.7$, corresponding to air. Our range of $\mathrm{Ro}^{-1}$ covered the regimes related to the turbomachinery, $R o^{-1} \sim O(1)$, and geophysical flows, $R o^{-1} \gg 1$.

The results show that below an optimal $R o_{o p t}^{-1}$, the Coriolis and buoyancy force interaction form a bidirectional wind. The value of $R o_{\text {opt }}^{-1}$ decreases from approximately 1.0 to 0.6 , as $R a$ increases from $10^{7}$ to $10^{10}$. At $R o_{o p t}^{-1}$, the wind momentum reaches its maximum, leading to a maximal $C_{f}$ and a minimal $N u$. Just beyond $R o_{o p t}^{-1}$, the Coriolis force locally dominates. It weakens the wind and forms a large-scale cyclone. At $R o^{-1} \gg R o_{o p t}^{-1}$, the Coriolis force fully dominates. It balances the pressure gradient (geostrophic balance), laminarises the flow and stabilises both cyclones and anticyclones. 
The flow regimes have both similarities and differences to those in rotating Rayleigh-Bénard convection. The differences are due to the different axis of rotation and boundary conditions. In rotating Rayleigh-Bénard convection, optimal transport $\left(R o_{o p t}^{-1}\right)$ occurs only if the hot and cold boundaries are no-slip (rot_RB_wall and rot_RB_cyl in table 1). The presence of the wall, hence the Ekman layer, forms coherent columnar or plume-like structures. These structures enhance vertical transport, leading to a maximal $N u$. On the other hand, large-scale quasi-two-dimensional cyclone has only been observed in rot_RB_slip and rot_RB_wall so far. At $R o^{-1} \gg 1$, the geostrophic regime occurs for all the rotating Rayleigh-Bénard systems, but the flow evolution depends on the boundary conditions.

Our study highlights that with centrifugal convection we can control the wind at $R o_{o p t}^{-1}$ to generate a shear boundary layer, providing the opportunity to hasten transition to turbulent. Turbulent shear boundary layer (Marusic et al. 2010) is the main assumption for the ultimate regime (Kraichnan 1962; Grossmann \& Lohse 2011). By our highest $R a=10^{10}$, we see transitional behaviour in the boundary layer. In particular, the boundary layer yields streak-like structures with a spectral distribution similar to a canonical turbulent boundary layer. Yet, the mean flow does not reach the Prandtl-von Kármán (logarithmic) behaviour. Recently, Iyer et al. (2020) performed Rayleigh-Bénard simulation up to $R a=10^{15}$ in a slender cylindrical cell of aspect ratio 1/10. They did not see a departure from the classical regime, because the boundary layer structure differed from a unidirectional canonical turbulent boundary layer. As they discuss, thermal plumes block the boundary layer from development. We also conjecture that the slender cylinder does not allow the boundary layer to develop.

From an experimental perspective, the bidirectional wind is a bonus to reach the ultimate regime, in addition to mitigating non-Oberbeck-Boussinesq effects. Experiments are conducted in a vertical cylindrical annulus with closed end walls. As a result, some additional phenomena may affect the flow, including gravitational buoyancy, Ekman and Stewartson layers (Jacoby et al. 2011; Pitz et al. 2017b; Von Larcher et al. 2018). However, these effects can be minimised by adjusting the working fluid, operating conditions and geometry. The gravitational buoyancy effect can be minimised by making $R \Omega^{2} \gg g$. For instance, Jiang et al. (2020) could experimentally achieve $R \Omega^{2} \simeq 60 g$. The Ekman layer effect can be minimised by making $L / H \gg E k^{-1 / 2}$ (Busse 1970), where $L$ is the annulus height and $E k \equiv v /\left(\Omega L^{2}\right)$ is the Ekman number. Busse \& Carrigan (1974) could experimentally mitigate the Ekman layer effect by making $L / H \simeq 50$. Alonso et al. (1999) show numerically that Ekman and Stewartson layer effects can be minimised by increasing $\operatorname{Pr}, R / H, L / R$ or Taylor number $T a \equiv\left(2 \Omega H^{2} / \nu\right)^{2} \equiv R a R o^{-2} / P r$. To our knowledge, most of the recent experiments consider $R, H$ and $L$ in the same order (Von Larcher \& Egbers 2005; Von Larcher et al. 2018; Jiang et al. 2020). Nevertheless, Jiang et al. (2020) show small differences in $N u$ between their experimental rig (closed cavity) and DNS (open ended shell). However, all the experiments so far operate at $R o^{-1}>2$ (figure $2 a$ ). Therefore, the effects of Ekman and Stewartson layers on the bidirectional wind (at $R o_{o p t}^{-1} \lesssim 1$ ) and how effective the mitigating prescriptions are remain to be investigated.

Answering the above questions lead us to several future directions. These directions also address some important aspects of turbomachinery studies. One direction is to enclose the boundaries normal to the rotation axis (closed cavity). Therefore, we can study the effects of Ekman and Stewartson layers on the bidirectional wind. Additionally, our system will be more similar to a compressor cavity (figure 1 in Owen \& Long (2015)). Another direction is to systematically change the ratios $R / H$ and $L / R$. Therefore, we can study how these geometrical parameters alter the interaction between the bidirectional wind and viscous layers. Also, we can evaluate the prescriptions (Busse 1970; Alonso et al. 
1999) for mitigating the effects of viscous layers. This direction is also valuable from a turbomachinery perspective. In compressor cavities (Atkins \& Kanjirakkad 2014), $H / R$ varies from approximately 0.9 (Farthing et al. 1992) to 0.5 (Bohn et al. 1995).

\section{Acknowledgements}

We acknowledge PRACE for awarding us access to JUWELS at the Jülich Supercomputing Centre under PRACE project number 2017174146. We also acknowledge the Pawsey Supercomputing Centre with funding from the Australian Government and the Government of Western Australia, and the National Computing Infrastructure (NCI), which is supported by the Australian Government. This research was partially supported by Natural Science Foundation of China under grant nos. 11988102, 91852202. The support of the Australian Research Council is also gratefully acknowledged. We thank the anonymous reviewers for their helpful comments which improved the quality of the manuscript.

\section{Declaration of interests}

The authors report no conflict of interest.

\section{Appendix A. Flow asymmetry beyond the optimal Coriolis force}

Slightly beyond $R o_{o p t}^{-1}$, the flow becomes asymmetric (e.g. figure $7 c, g$ ). This asymmetry occurs at all values of $R a=10^{7}-10^{10}$ (the marked profiles in figure 11). To assess the persistence of this asymmetry, we simulated the case at $R a=10^{8}, R o^{-1}=1$ (figure $7 c, g$ ) for $1200 H / U$ (figure 19, simulation A). Simulation A is initialised with a large cyclone in the bulk (see the leftmost field in figure 19). But it converges to an asymmetric field with a cyclone near the top wall. The simulation time of $1200 \mathrm{H} / \mathrm{U}$ is approximately four times that of the one required for Rayleigh-Bénard convection (Stevens et al. 2010). We also performed simulation B by inverting $\mathrm{A}$ at the last time-step $t_{N}: u_{B}(x, y, z, 0)=u_{A}\left(x, y, H-z, t_{N}\right), v_{B}(x, y, z, 0)=v_{A}(x, y, H-$ $\left.z, t_{N}\right), w_{B}(x, y, z, 0)=-w_{A}\left(x, y, H-z, t_{N}\right), \theta_{B}(x, y, z, 0)=-\theta_{A}\left(x, y, H-z, t_{N}\right)$. With this inversion, the initial condition for B corresponds to an anticlockwise rotating system, opposite to the rotation direction during the simulation (figure 19). We simulated B for approximately $1200 \mathrm{H} / \mathrm{U}$. The simulation converges to the same flow field as A, with equal $N u$ and $C_{f}$. Therefore, vertical asymmetry is the converged solution at $R a=10^{8}$ and $R o^{-1}=1.0$.

Flow asymmetry is possible if the Coriolis force in $(2.1 a)-(2.1 e)$ is asymmetric. Slightly beyond $R o_{o p t}^{-1}$, the Coriolis force is dominant either near the top wall (figure $7 c, g$ ) or bottom wall (figure 12a). The dominant Coriolis force dampens $\overline{u^{\prime} w^{\prime}}$ on one side of the domain (e.g. solid black line in figure $8 c$ ). According to $(3.1 a, b)$, the mean velocity profile depends on $\overline{u^{\prime} w^{\prime}}$. Therefore, the asymmetry in $\overline{u^{\prime} w^{\prime}}$ causes the asymmetry in $\bar{u}$. The flow asymmetry also occurs in spanwise rotating channel flow (Kristoffersen \& Andersson 1993; Brethouwer 2016, 2017). Similar to here, the asymmetric Coriolis force suppresses $\overline{u^{\prime} w^{\prime}}$ on one side of the channel.

\section{Appendix B. Centrifugal convection with wall versus slip boundaries}

Our system in the present study (figure 1) is centrifugal convection with no-slip hot and cold boundaries (CC_wall in table 1). von Hardenberg et al. (2015) considered centrifugal 
(a)
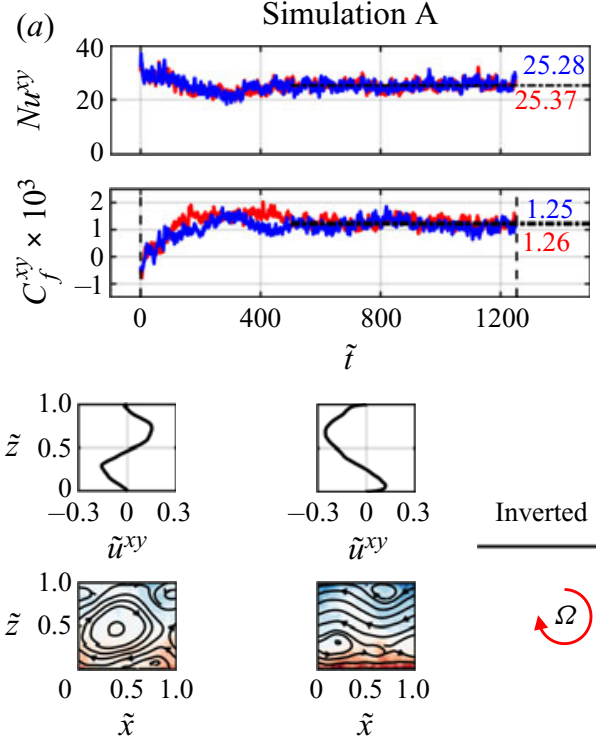
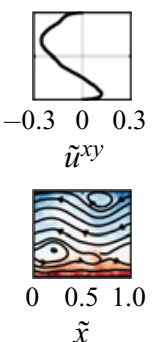

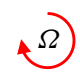

(b)
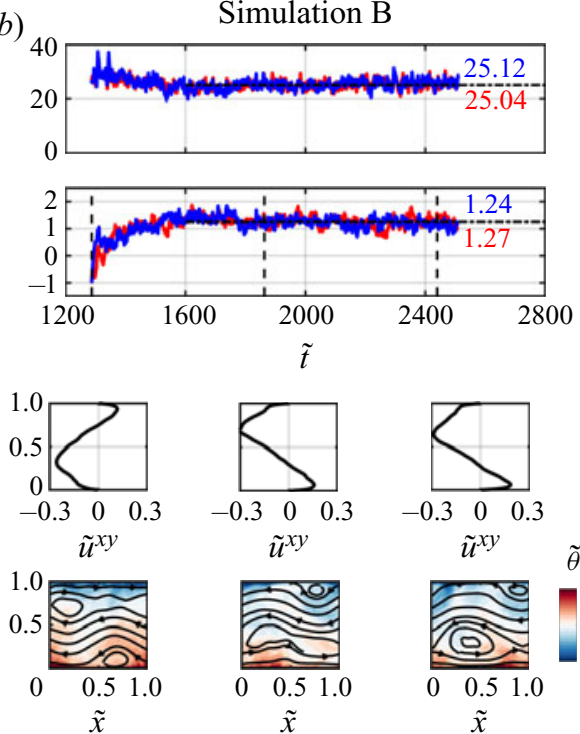

FIgURE 19. Comparison of simulation A $(a)$ with B (b) for $R a=10^{8}$ and $R o^{-1}=1$. To initialise $\mathrm{B}$, we invert $\mathrm{A}$ at the last time-step $t_{N}: u_{B}(x, y, z, 0)=u_{A}\left(x, y, H-z, t_{N}\right)$, $v_{B}(x, y, z, 0)=v_{A}\left(x, y, H-z, t_{N}\right), \quad w_{B}(x, y, z, 0)=-w_{A}\left(x, y, H-z, t_{N}\right), \quad \theta_{B}(x, y, z, 0)=$ $-\theta_{A}\left(x, y, H-z, t_{N}\right)$. The first and second rows from the top are the history of plane averaged $N u^{x y}$ and $C_{f}^{x y}$, respectively, at the bottom wall (solid red line) and top wall (solid blue line). Here $\tilde{t} \equiv t U / H$ is the normalised simulation time. The horizontal dashed-dotted lines locate the time-averaged $N u^{x y}$ and $C_{f}^{x y}$ over their converged period; the averaged values are reported next to each line. The third row is the plane-averaged $\tilde{u}^{x y} \equiv u^{x y} / U$ at the times identified with vertical dashed line in the $C_{f}^{x y}$ history. The fourth row shows the flow fields at the same times. They show the spanwise averaged $\tilde{\theta}^{y} \equiv \theta^{y} / \Delta$ overlaid by the streamlines of $\left(\tilde{u}^{y}, \tilde{w}^{y}\right) \equiv\left(u^{y} / U, w^{y} / U\right)$. The circled red arrow indicates the system rotation direction (clockwise).
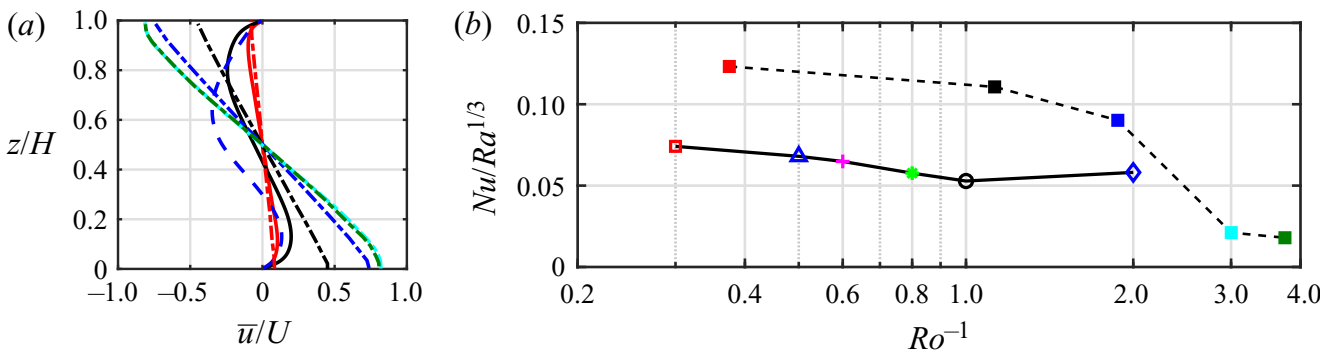

FIGURE 20. Comparison between centrifugal convection with slip (CC_slip, von Hardenberg et al. (2015)) and no-slip (CC_wall, present data) hot and cold boundaries at $R a=10^{7}$ and $0.3 \lesssim R o^{-1} \lesssim 4$.0. (a) The $\bar{u}$ profiles for CC_slip at $R o^{-1} \simeq 0.38$ (dashed-dotted red line), 1.13 (dashed-dotted black line), 1.88 (dashed-dotted blue line), 3.00 (dashed-dotted cyan line) and 3.75 (dashed-dotted forest green line), and $\mathrm{CC}_{-}$wall at $\mathrm{Ro}^{-1}=0.3$ (solid red line), 1.0 (solid black line) and 2.0 (dashed blue line). (b) The $N u$ for CC_slip (dashed black line) at $R o^{-1} \simeq 0.38$ (filled red square), 1.13 (filled black square), 1.88 (filled blue square), 3.00 (filled cyan square) and 3.75 (filled forest green square), and CC_wall (solid black line) at $R o^{-1}=0.3$ ( $\square$, red), 0.5 $(\triangle$, blue $), 0.6(+$, magenta), $0.8(*$, green $), 1.0(\circ$, black) and $2.0(\diamond$, blue) 
convection with free-slip hot and cold boundaries (CC_slip in table 1). We compare these two systems in terms of $\bar{u}$ (figure 20a) and $N u$ (figure 20b). We consider $P r \simeq$ $0.7, R a=10^{7}$ and $0.3 \lesssim R o^{-1} \lesssim 4.0$, where there is an overlap between our parameter space and the one by von Hardenberg et al. (2015) ( $\circ$, black; $\nabla$, red in figure $2 a$ ). In CC_slip, the bidirectional wind can have a cyclonic or anticyclonic mean vorticity (von Hardenberg et al. 2015). Here, we only consider the anticyclonic wind, because in CC_wall the bidirectional wind is always anticyclonic. In CC_slip (dashed-dotted lines in figure 20a), the bidirectional wind never breaks down. Its strength increases up to $R O^{-1} \simeq 3.0$ (dashed-dotted cyan line); beyond $R O^{-1} \simeq 3.0$ (dashed-dotted forest green line) the wind strength saturates. In CC_wall (solid lines in figure 20a), the wind strength increases up to $R o_{o p t}^{-1} \simeq 1$ (solid black line); beyond $R o_{o p t}^{-1}$ (dashed blue line) the wind breaks down.

The behaviour in $\mathrm{Nu}$ (figure $20 \mathrm{~b}$ ) is consistent with the evolution of wind structure. In CC_slip (dashed black line in figure $20 b$ ), $N u$ monotonically decreases up to $R o^{-1} \simeq 3.0$. Because the bidirectional wind increases in strength. Beyond $R o^{-1} \simeq 3.0, N u$ does not change because the bidirectional wind does not change. In CC_wall (solid black line in figure 20b), $N u$ decreases up to $R o_{\text {opt }}^{-1} \simeq 1$, because the bidirectional wind increases in strength. Beyond $R o_{o p t}^{-1} \simeq 1, N u$ increases because the bidirectional wind breaks down.

\section{REFERENCES}

Aguirre Guzmán, A. J., Madonia, M., Cheng, J. S., Ostilla-Mónico, R., Clercx, H. J. H. \& KUNNEN, R. P. J. 2020 Frictional boundary layer effect on vortex condensation in rotating turbulent convection. arXiv:2001.11889.

Ahlers, G., Grossmann, S. \& Lohse, D. 2009 Heat transfer and large scale dynamics in turbulent Rayleigh-Bénard convection. Rev. Mod. Phys. 81, 503-537.

Ahlers, G., He, X., Funfschilling, D. \& Bodenschatz, E. G. 2012 Heat transport by turbulent Rayleigh-Bénard convection for $P r \simeq 0.8$ and $3 \times 10^{12} \lesssim R a \lesssim 10^{15}$ : aspect ratio $\Gamma=0.50$. New J. Phys. 14, 103012.

Alonso, A., Net, M., Mercader, I. \& Knobloch, E. 1999 Onset of convection in a rotating annulus with radial gravity and heating. Fluid Dyn. Res. 24, 133.

AtKins, N. R. \& KANJIRAKKAD, V. 2014 Flow in a rotating cavity with axial throughflow at engine representative conditions. In Proceedings of ASME Turbo Expo 2014: Turbine Technical Conference and Exposition. American Society of Mechanical Engineers.

Bartello, P., Métais, O. \& Lesieur, M. 1994 Coherent structures in rotating three-dimensional turbulence. J. Fluid Mech. 273, 1-29.

Blass, A., Zhu, X., Verzicco, R., Lohse, D. \& Stevens, R. J. A. M. 2020 Flow organization and heat transfer in turbulent wall sheared thermal convection. J. Fluid Mech. 897, A22.

Bohn, D., Deuker, E., Emunds, R. \& Gorzelitz, V. 1995 Experimental and theoretical investigations of heat transfer in closed gas-filled rotating annuli. Trans. ASME: J. Turbomach. 117, 175-183.

BRETHOUWER, G. 2016 Linear instabilities and recurring bursts of turbulence in rotating channel flow simulations. Phys. Rev. Fluids 1 (5), 054404.

Brethouwer, G. 2017 Statistics and structure of spanwise rotating turbulent channel flow at moderate Reynolds numbers. J. Fluid Mech. 828, 424-458.

Busse, F. H. 1970 Thermal instabilities in rapidly rotating systems. J. Fluid Mech. 44, 441-460.

Busse, F. H. \& CARrigan, C. R. 1974 Convection induced by centrifugal buoyancy. J. Fluid Mech. 62, 579-592.

Calzavarini, E., Lohse, D., Toschi, F. \& Tripiccione, R. 2005 Rayleigh and Prandtl number scaling in the bulk of Rayleigh-Bénard turbulence. Phys. Fluids 17, 055107. 
Cheng, J. S., Stellmach, S., Ribeiro, A., Grannan, A., King, E. M. \& Aurnou, J. M. 2015 Laboratory-numerical models of rapidly rotating convection in planetary cores. Geophys. J. Intl 201, 1-17.

Chong, K. L., Huang, S. D., Kaczorowski, M. \& Xia, K. Q. 2015 Condensation of coherent structures in turbulent flows. Phys. Rev. Lett. 115, 264503.

Chong, K. L., Yang, Y., Huang, S. D., Zhong, J. Q., Stevens, R. J., Verzicco, R., Lohse, D. \& XIA, K. Q. 2017 Confined Rayleigh-Bénard, rotating Rayleigh-Bénard, and double diffusive convection: A unifying view on turbulent transport enhancement through coherent structure manipulation. Phys. Rev. Lett. 119, 064501.

DEARDORFF, J. W. 1970 Convective velocity and temperature scales for the unstable planetary boundary layer and for Rayleigh convection. J. Atmos. Sci. 27, 1211-1213.

Deremble, B., Johnson, E. R. \& Dewar, W. K. 2017 A coupled model of interior balanced and boundary flow. Ocean Model. 119, 1-12.

Dritschel, D. G., Reinaud, J. N. \& MCKiver, W. J. 2004 The quasi-geostrophic ellipsoidal vortex model. J. Fluid Mech. 505, 201-223.

ECKE, R. E. 2015 Scaling of heat transport near onset in rapidly rotating convection. Phys. Lett. A 379, 2221-2223.

ECKE, R. E. \& NiEMELA, J. J. 2014 Heat transport in the geostrophic regime of rotating Rayleigh-Bénard convection. Phys. Rev. Lett. 113 (11), 114301.

Farthing, P. R., Long, C. A., Owen, J. M. \& Pincombe, J. R. 1992 Rotating cavity with axial throughflow of cooling air: flow structure. Trans. ASME: J. Turbomach. 114, 237-246.

FAVIER, B. \& KNOBLOCH, E. 2020 Robust wall states in rapidly rotating Rayleigh-Bénard convection. J. Fluid Mech. 895, R1.

Favier, B., Silvers, L. J. \& Proctor, M. R. E. 2014 Inverse cascade and symmetry breaking in rapidly rotating Boussinesq convection. Phys. Fluids 26, 096605.

Fowlis, W. W. \& HidE, R. 1965 Thermal convection in a rotating annulus of liquid: effect of viscosity on the transition between axisymmetric and non-axisymmetric flow regimes. J. Atmos. Sci. 22, 541-558.

GODEFERD, F. S. \& LOLLINI, L. 1999 Direct numerical simulations of turbulence with confinement and rotation. J. Fluid Mech. 393, 257-308.

Greenspan, H. P. 1968 The Theory of Rotating Fluids. Cambridge University Press.

Grossmann, S. \& LohSE, D. 2000 Scaling in thermal convection: a unifying theory. J. Fluid Mech. $407,27-56$.

Grossmann, S. \& LoHSE, D. 2011 Multiple scaling in the ultimate regime of thermal convection. Phys. Fluids 23, 045108.

Grossmann, S., LOHSE, D. \& Sun, C. 2016 High-Reynolds number Taylor-Couette turbulence. Annu. Rev. Fluid Mech. 48, 53-80.

GRÖtZBACH, G. 1983 Spatial resolution requirements for direct numerical simulation of the Rayleigh-Bénard convection. J. Comput. Phys. 49, 241-264.

Guervilly, C., Hughes, D. W. \& Jones, C. A. 2014 Large-scale vortices in rapidly rotating Rayleigh-Bénard convection. J. Fluid Mech. 758, 407-435.

von Hardenberg, J., Goluskin, D., Provenzale, A. \& Spiegel, E. A. 2015 Generation of large-scale winds in horizontally anisotropic convection. Phys. Rev. Lett. 115, 134501.

He, X., Bodenschatz, E. \& Ahlers, G. 2016 Azimuthal diffusion of the large-scale-circulation plane, and absence of significant non-boussinesq effects, in turbulent convection near the ultimate-state transition. J. Fluid Mech. 791, R3.

He, X., Funfschilling, D., Bodenschatz, E. \& Ahlers, G. $2012 a$ Heat transport by turbulent Rayleigh-Bénard convection for $\operatorname{Pr} \simeq 0.8$ and $4 \times 10^{11} \lesssim R a \lesssim 2 \times 10^{14}$ : ultimate-state transition for aspect ratio $\Gamma=1.00$. New J. Phys. 14, 063030.

He, X., Funfschilling, D., Nobach, H., Bodenschatz, E. \& Ahlers, G. $2012 b$ Transition to the ultimate state of turbulent Rayleigh-Bénard convection. Phys. Rev. Lett. 108, 024502.

He, X., Funfschilling, D., Nobach, H., Bodenschatz, E. \& Ahlers, G. 2013 Comment on "effect of boundary layers asymmetry on heat transfer efficiency in turbulent Rayleigh-Bénard convection at very high Rayleigh numbers". Phys. Rev. Lett. 110, 199401. 
Hide, R. \& MAson, P. J. 1970 Baroclinic waves in a rotating fluid subject to internal heating. Phil. Trans. R. Soc. Lond. A 268, 201-232.

Hopfinger, E. J., BRowAnd, F. K. \& GAGNe, Y. 1982 Turbulence and waves in a rotating tank. J. Fluid Mech. 125, 505-534.

HoRn, S. \& SHISHKinA, O. 2014 Rotating non-oberbeck-boussinesq Rayleigh-Bénard convection in water. Phys. Fluids 26, 055111.

HORN, S. \& SHISHKINA, O. 2015 Toroidal and poloidal energy in rotating Rayleigh-Bénard convection. J. Fluid Mech. 762, 232-255.

Iyer, K. P., Scheel, J. D., Schumacher, J. \& SReenivasan, K. R. 2020 Classical 1/3 scaling of convection holds up to Ra $=10^{15}$. Proc. Natl Acad. Sci. USA 117, 7594-7598.

JaCOBY, T. N. L., READ, P. L., Williams, P. D. \& YounG, R. M. B. 2011 Generation of inertia-gravity waves in the rotating thermal annulus by a localised boundary layer instability. Geophys. Astrophys. Fluid Dyn. 105, 161-181.

JiAnG, H., Zhu, X., WANG, D., Huisman, S. G. \& Sun, C. 2020 Supergravitational turbulent thermal convection. Sci. Adv. 6, eabb8676.

Julien, K., LegG, S., MCWilliams, J. \& Werne, J. $1996 a$ Hard turbulence in rotating Rayleigh-Bénard convection. Phys. Rev. E 53, R5557.

Julien, K., LegG, S., MCWilliams, J. \& Werne, J. $1996 b$ Rapidly rotating turbulent Rayleigh-Bénard convection. J. Fluid Mech. 322, 243-273.

Julien, K., LegG, S., McWilliams, J. \& Werne, J. 1999 Plumes in rotating convection. Part 1. Ensemble statistics and dynamical balances. J. Fluid Mech. 391, 151-187.

Julien, K., Rubio, A. M, Grooms, I. \& Knobloch, E. 2012 Statistical and physical balances in low Rossby number Rayleigh-Bénard convection. Geophys. Astrophys. Fluid Dyn. 106, 392-428.

KIM, J. \& MoIN, P. 1989 Transport of passive scalars in a turbulent channel flow. In Turbulent Shear Flows (ed. J.-C. André et al.), vol. 6, pp. 85-96. Springer.

King, E. M., Stellmach, S. \& AURnOU, J. M. 2012 Heat transfer by rapidly rotating Rayleigh-Bénard convection. J. Fluid Mech. 691, 568-582.

King, E. M., Stellmach, S., Noir, J., Hansen, U. \& Aurnou, J. M. 2009 Boundary layer control of rotating convection systems. Nature 457, 301 .

KING, M. P., WiLson, M. \& OWEN, J. M. 2007 Rayleigh-Bénard convection in open and closed rotating cavities. Trans. ASME: J. Engng Gas Turbines Power 129, 305-311.

Kinzel, M., Wolf, M., Holzner, M., Lüthi, B., Tropea, C. \& Kinzelbach, W. 2011 Simultaneous two-scale 3D-PTV measurements in turbulence under the influence of system rotation. Exp. Fluids 51, 75-82.

Kline, S. J., Reynolds, W. C., Schraub, F. A. \& Runstadler, P. W. 1967 The structure of turbulent boundary layers. J. Fluid Mech. 30, 741-773.

Kraichnan, R. H. 1962 Turbulent thermal convection at arbitrary Prandtl number. Phys. Fluids 5, 1374-1389.

KRISTOFFERSEN, R. \& ANDERSSON, H. I. 1993 Direct simulations of low-Reynolds-number turbulent flow in a rotating channel. J. Fluid Mech. 256, 163-197.

Kundu, P. K. \& Cohen, L. M. 1990 Fluid mechanics, 2nd edn. Academic Press.

Kunnen, R. P. J., Clercx, H. J. H. \& Geurts, B. J. 2006 Heat flux intensification by vortical flow localization in rotating convection. Phys. Rev. E 74, 056306.

Kunnen, R. P. J., Clercx, H. J. H. \& Geurts, B. J. 2008 Breakdown of large-scale circulation in turbulent rotating convection. Europhys. Lett. 84, 24001.

Kunnen, R. P. J., Clercx, H. J. H. \& Geurts, B. J. $2010 a$ Vortex statistics in turbulent rotating convection. Phys. Rev. E 82, 036306.

Kunnen, R. P. J., Geurts, B. J. \& ClercX, H. J. H. 2009 Turbulence statistics and energy budget in rotating Rayleigh-Bénard convection. Eur. J. Mech. B/Fluids 28, 578-589.

Kunnen, R. P. J., Geurts, B. J. \& ClercX, H. J. H. $2010 b$ Experimental and numerical investigation of turbulent convection in a rotating cylinder. J. Fluid Mech. 642, 445-476.

Kunnen, R. P. J., Ostilla-Mónico, R., VAn der Poel, E. P., Verzicco, R. \& Lohse, D. 2016 Transition to geostrophic convection: the role of the boundary conditions. J. Fluid Mech. 799, 413-432. 
Kunnen, R. P. J., Stevens, R. J. A. M., Overkamp, J., Sun, C., van Heijst, G. F. \& ClercX, H. J. H. 2011 The role of Stewartson and Ekman layers in turbulent rotating Rayleigh-Bénard convection. J, Fluid Mech. 688, 422-442.

Lee, M. \& Moser, R. D. 2015 Direct numerical simulation of turbulent channel flow up to $\operatorname{Re}_{\tau} \approx 5200$. J. Fluid Mech. 774, 395-415.

Lim, Z. L., Chong, K. L., Ding, G. Y. \& XIA, K. Q. 2019 Quasistatic magnetoconvection: heat transport enhancement and boundary layer crossing. J. Fluid Mech. 870, 519-542.

LIU, Y. \& ECKE, R. E. 2009 Heat transport measurements in turbulent rotating Rayleigh-Bénard convection. Phys. Rev. E 80, 036314.

Marusic, I., McKeon, B. J., Monkewitz, P. A., Nagib, H. M., Smits, A. J. \& SREEnivASAN, K. R. 2010 Wall-bounded turbulent flows at high Reynolds numbers: recent advances and key issues. Phys. Fluids 22, 065103.

Moisy, F., Morize, C., RABAud, M. \& Sommeria, J. 2011 Decay laws, anisotropy and cyclone-anticyclone asymmetry in decaying rotating turbulence. J. Fluid Mech. 666, 5-35.

Morize, C., Moisy, F. R. \& RABAUd, M. 2005 Decaying grid-generated turbulence in a rotating tank. Phys. Fluids 17, 095105.

NG, C. S., Ooi, A., Lohse, D. \& Chung, D. 2015 Vertical natural convection: application of the unifying theory of thermal convection. J. Fluid Mech. 764, 349-361.

NG, C. S., Ooi, A., Lohse, D. \& Chung, D. 2017 Changes in the boundary-layer structure at the edge of the ultimate regime in vertical natural convection. J. Fluid Mech. 825, 550-572.

NG, C. S., Ooi, A., Lohse, D. \& Chung, D. 2018 Bulk scaling in wall-bounded and homogeneous vertical natural convection. J. Fluid Mech. 841, 825-850.

Novi, L., von Hardenberg, J., Hughes, D. W., Provenzale, A. \& Spiegel, E. A. 2019 Rapidly rotating Rayleigh-Bénard convection with a tilted axis. Phys. Rev. E 99, 053116.

Owen, J. M. \& Long, C. A. 2015 Review of buoyancy-induced flow in rotating cavities. Trans. ASME: J. Turbomach. 137, 111001.

Pfeffer, R. L., Fowlis, W. W., Fein, J. \& Buckley, J. 1970 Experimental determinations of the transition between the symmetrical and wave regimes in a rotating differentially heated annulus of fluid. Pure Appl. Geophys. 81, 263-271.

Pieri, A. B., Falasca, F., von Hardenberg, J. \& Provenzale, A. 2016 Plume dynamics in rotating Rayleigh-Bénard convection. Phys. Lett. A 380, 1363-1367.

Pirozzoli, S., Bernardini, M., Verzicco, R. \& Orlandi, P. 2017 Mixed convection in turbulent channels with unstable stratification. J. Fluid Mech. 821, 482-516.

Pitz, D. B., Chew, J. W. \& MARXEN, O. 2019 Large-eddy simulation of buoyancy-induced flow in a sealed rotating cavity. Trans. ASME: J. Engng Gas Turbines Power 141, 021020.

Pitz, D. B., Chew, J. W., Marxen, O. \& Hills, N. J. $2017 a$ Direct numerical simulation of rotating cavity flows using a spectral element-fourier method. Trans. ASME: J. Engng Gas Turbines Power 139, 072602 .

Pitz, D. B., MARXen, O. \& CHEW, J. W. $2017 b$ Onset of convection induced by centrifugal buoyancy in a rotating cavity. J. Fluid Mech. 826, 484-502.

Rajaei, H., Kunnen, R. P. J. \& ClercX, H. J. H. 2017 Exploring the geostrophic regime of rapidly rotating convection with experiments. Phys. Fluids 29, 045105.

RAndriamampianina, A., FrüH, W. G., ReAd, P. L. \& MAubert, P. 2006 Direct numerical simulations of bifurcations in an air-filled rotating baroclinic annulus. J. Fluid Mech. 561, 359-389.

READ, P. L. 2001 Transition to geostrophic turbulence in the laboratory, and as a paradigm in atmospheres and oceans. Surv. Geophys. 22, 265-317.

Read, P. L., Collins, M., FrüH, W. G., Lewis, S. R. \& Lovegrove, A. F. 1998 Wave interactions and baroclinic chaos: a paradigm for long timescale variability in planetary atmospheres. Chaos, Solitons Fractals 9, 231-249.

Read, P. L., Maubert, P., Randriamampianina, A. \& FrüH, W. G. 2008 Direct numerical simulation of transitions towards structural vacillation in an air-filled, rotating, baroclinic annulus. Phys. Fluids 20, 044107.

ReInAUd, J. N. \& CARTON, X. 2016 The interaction between two oppositely travelling, horizontally offset, antisymmetric quasi-geostrophic hetons. J. Fluid Mech. 794, 409-443. 
Reinaud, J. N., DRitschel, D. G. \& Koudella, C. R. 2003 The shape of vortices in quasi-geostrophic turbulence. J. Fluid Mech. 474, 175-192.

Roche, P. E., Gauthier, F., Chabaud, B. \& Hébral, B. 2005 Ultimate regime of convection: robustness to poor thermal reservoirs. Phys. Fluids 17, 115107.

SCHEEL, J. D. \& SCHUMACHER, J. 2016 Global and local statistics in turbulent convection at low Prandtl numbers. J. Fluid Mech. 802, 147-173.

SChmitz, S. \& Tilgner, A. 2009 Heat transport in rotating convection without Ekman layers. Phys. Rev. E 80, 015305.

Schmitz, S. \& Tilgner, A. 2010 Transitions in turbulent rotating Rayleigh-Bénard convection. Geophys. Astrophys. Fluid Dyn. 104, 481-489.

SHIsHKINA, O. 2020 Tenacious wall states in thermal convection in rapidly rotating containers. J. Fluid Mech. 898, F1.

Shishrina, O., Stevens, R. J. A. M., Grossmann, S. \& Lohse, D. 2010 Boundary layer structure in turbulent thermal convection and its consequences for the required numerical resolution. New J. Phys. 12, 075022.

SMith, L. M. \& LEE, Y. 2005 On near resonances and symmetry breaking in forced rotating flows at moderate Rossby number. J. Fluid Mech. 535, 111-142.

SMith, L. M. \& WALEFFE, F. 1999 Transfer of energy to two-dimensional large scales in forced, rotating three-dimensional turbulence. Phys. Fluids 11, 1608-1622.

Stellmach, S., Lischper, M., Julien, K., Vasil, G., Cheng, J. S., Ribeiro, A., King, E. M. \& AURNOU, J. M. 2014 Approaching the asymptotic regime of rapidly rotating convection: boundary layers versus interior dynamics. Phys. Rev. Lett. 113, 254501.

Stevens, R. J. A. M., Blass, A., Zhu, X., Verzicco, R. \& Lohse, D. 2018 Turbulent thermal superstructures in Rayleigh-Bénard convection. Phys. Rev. Fluids 3, 041501.

Stevens, R. J. A. M., ClercX, H. J. H. \& Lohse, D. 2012 Breakdown of the large-scale circulation in $\gamma=1 / 2$ rotating Rayleigh-Bénard flow. Phys. Rev. E 86, 056311.

Stevens, R. J. A. M., ClercX, H. J. H. \& Lohse, D. $2013 a$ Heat transport and flow structure in rotating Rayleigh-Bénard convection. Eur. J. Mech. B/Fluids 40, 41-49.

Stevens, R. J. A. M., Overkamp, J., Lohse, D. \& ClercX, H. J. H. 2011 Effect of aspect ratio on vortex distribution and heat transfer in rotating Rayleigh-Bénard convection. Phys. Rev. E 84, 056313.

Stevens, R. J. A. M., van der Poel, E. P., Grossmann, S. \& Lohse, D. $2013 b$ The unifying theory of scaling in thermal convection: the updated prefactors. J. Fluid Mech. 730, 295-308.

Stevens, R. J. A. M., Verzicco, R. \& Lohse, D. 2010 Radial boundary layer structure and Nusselt number in Rayleigh-Bénard convection. J. Fluid Mech. 643, 495-507.

Stevens, R. J. A. M., Zhong, J. Q., Clercx, H. J. H., Ahlers, G. \& Lohse, D. 2009 Transitions between turbulent states in rotating Rayleigh-Bénard convection. Phys. Rev. Lett. 103, 024503.

Sugihara, Y., Migita, M. \& Honji, H. 2005 Orderly flow structures in grid-generated turbulence with background rotation. Fluid Dyn. Res. 36, 23.

VeNAILle, A. 2020 Quasi-geostrophy against the wall. J. Fluid Mech. 894, R1.

VON LARChER, T. \& DÖRnBRACK, A. 2014 Numerical simulations of baroclinic driven flows in a thermally driven rotating annulus using the immersed boundary method. Meteorol. Z. 23, 599-610.

VON LARCHER, T. H. \& EgBers, C. 2005 Experiments on transitions of baroclinic waves in a differentially heated rotating annulus. Nonlinear Process. Geophys. 12, 1033-1041.

Von Larcher, T., Viazzo, S., Harlander, U., Vincze, M. \& Randriamampianina, A. 2018 Instabilities and small-scale waves within the Stewartson layers of a thermally driven rotating annulus. J. Fluid Mech. 841, 380-407.

VorobiefF, P. \& ECKE, R. E. 2002 Turbulent rotating convection: an experimental study. J. Fluid Mech. 458, 191-218.

Weiss, S. \& AHLERS, G. 2011a Heat transport by turbulent rotating Rayleigh-Bénard convection and its dependence on the aspect ratio. J. Fluid Mech. 684, 407-426.

Weiss, S. \& AHLERs, G. $2011 b$ The large-scale flow structure in turbulent rotating Rayleigh-Bénard convection. J. Fluid Mech. 688, 461-492. 
Weiss, S., Stevens, R. J. A. M., Zhong, J. Q., Clercx, H. J. H., Lohse, D. \& Ahlers, G. 2010 Finite-size effects lead to supercritical bifurcations in turbulent rotating Rayleigh-Bénard convection. Phys. Rev. Lett. 105, 224501.

Weiss, S., WeI, P. \& Ahlers, G. 2016 Heat-transport enhancement in rotating turbulent Rayleigh-Bénard convection. Phys. Rev. E 93, 043102.

de Wit, X. M., Aguirre Guzmán, A. J., Madonia, M., Cheng, J. S., Clercx, H. J. H. \& Kunnen, R. P. J. 2020 Turbulent rotating convection confined in a slender cylinder: the sidewall circulation. Phys. Rev. Fluids 5, 023502.

XIE, Y. C., HU, Y. B. \& XIA, K. Q. 2019 Universal fluctuations in the bulk of Rayleigh-Bénard turbulence. J. Fluid Mech. 878, R1.

YAGLOM, A. M. 1979 Similarity laws for constant-pressure and pressure-gradient turbulent wall flows. Annu. Rev. Fluid Mech. 11, 505-540.

YANG, Y., Verzicco, R. \& LOHSE, D. 2016 From convection rolls to finger convection in double-diffusive turbulence. Proc. Natl Acad. Sci. USA 113, 69-73.

Zhang, X., Van Gils, D. P. M., Horn, S., Wedi, M., Zwirner, L., Ahlers, G., Ecke, R. E., Weiss, S., Bodenschatz, E. \& Shishkina, O. 2020 Boundary zonal flow in rotating turbulent Rayleigh-Bénard convection. Phys. Rev. Lett. 124, 084505.

ZHONG, J. Q. \& AhLERs, G. 2010 Heat transport and the large-scale circulation in rotating turbulent Rayleigh-Bénard convection. J. Fluid Mech. 665, 300-333.

ZhONG, J. Q., LI, H. M. \& WANG, X. Y. 2017 Enhanced azimuthal rotation of the large-scale flow through stochastic cessations in turbulent rotating convection with large Rossby numbers. Phys. Rev. Fluids 2, 044602.

Zhong, J. Q., Stevens, R. J. A. M., Clercx, H. J. H., Verzicco, R., Lohse, D. \& Ahlers, G. 2009 Prandtl-, Rayleigh-, and Rossby-number dependence of heat transport in turbulent rotating Rayleigh-Bénard convection. Phys. Rev. Lett. 102, 044502.

Zhou, Q., LiU, B. F., LI, C. M. \& ZHONG, B. C. 2012 Aspect ratio dependence of heat transport by turbulent Rayleigh-Bénard convection in rectangular cells. J. Fluid Mech. 710, 260-276.

Zhu, X., Varghese, M., Stevens, R. J. A. M., Verzicco, R. \& Lohse, D. 2018 Transition to the ultimate regime in two-dimensional Rayleigh-Bénard convection. Phys. Rev. Lett. 120, 144502. 